\title{
CFC+: improved dynamics and gravitational waveforms from relativistic core collapse simulations
}

\author{
P. Cerdá-Durán ${ }^{1}$, G. Faye ${ }^{2,4}$, H. Dimmelmeier ${ }^{3}$, J. A. Font ${ }^{1}$, \\ J. M. Ibáñez ${ }^{1}$ E. Müller ${ }^{3}$, and G. Schäfer ${ }^{2}$
}

${ }^{1}$ Departamento de Astronomía y Astrofísica, Universidad de Valencia, Dr. Moliner, 50, 46100 Burjassot, Valencia, Spain e-mail: pablo.cerda@uv.es

2 Theoretisch-Physikalisches Institut, Friedrich-Schiller-Universität Jena, Max-Wien-Platz 1, 07743 Jena, Germany

3 Max-Planck-Institut für Astrophysik, Karl-Schwarzschild-Str. 1, 85741 Garching, Germany

${ }^{4}$ Institut d'Astrophysique de Paris, 98bis boulevard Arago, 75014 Paris, France

Received 23 December 2004 / Accepted 24 January 2005

\begin{abstract}
Core collapse supernovae are a promising source of detectable gravitational waves. Most of the existing (multidimensional) numerical simulations of core collapse in general relativity were done using approximations of the Einstein field equations. As recently shown by Dimmelmeier et al. (2002a, A\&A, 388, 917), Dimmelmeier et al. (2002b, A\&A, 393, 523), one of the most interesting such approximations is the so-called conformal flatness condition (CFC) of Isenberg, Wilson; and Mathews. Building on this previous work we present new results from numerical simulations of relativistic rotational core collapse in axisymmetry, with the aim of improving the dynamics and gravitational waveforms. The computer code used for these simulations models the evolution of the coupled system of metric and fluid equations using the $3+1$ formalism, specialized to a new framework for the gravitational field equations we call CFC+. In this approach we add new degrees of freedom to the original CFC equations, which extend them by terms of second post-Newtonian order. The resulting metric equations are still of elliptic type, but the number of equations is significantly augmented in comparison to the original CFC approach. The hydrodynamic evolution and the CFC spacetime metric are calculated with the code developed by Dimmelmeier et al. (2002a, A\&A, 388, 917), which has been conveniently extended to account for the additional CFC+ equations. The corrections included in CFC+ are computed by solving a system of elliptic linear equations. The new formalism is assessed with time evolutions of both rotating neutron stars in equilibrium and gravitational core collapse of rotating polytropes. Gravitational wave signals for a comprehensive sample of collapse models are extracted using either the quadrupole formula or directly from the metric. We discuss our results on the dynamics and the gravitational wave emission through a detailed comparison between CFC and $\mathrm{CFC}+$ simulations. The main conclusion is that, for the neutron star spacetimes analyzed in the present work, no significant differences are found among $\mathrm{CFC}, \mathrm{CFC}+$, and full general relativity, which highlights the suitability of the former.
\end{abstract}

Key words. gravitation - gravitational waves - hydrodynamics - methods: numerical - relativity - stars: supernovae: general

\section{Introduction}

One of the most interesting topics in relativistic astrophysics is the formation and evolution of compact objects, such as those produced in the rotational core collapse of massive stars or produced in the merging of a compact binary consisting of two white dwarfs, of two neutron stars, or of a white dwarf and a neutron star. The catastrophic events in which the newborn compact objects are formed offer a promising source of gravitational waves in the $\mathrm{kHz}$ frequency range, thus optimal for detection of objects located at galactic distances with the new ground-based interferometer detectors such as LIGO or VIRGO.

Gravitational radiation is produced by accelerating matter whose dynamics is not spherically symmetric. Among astrophysical sources of gravitational radiation, axisymmetric sources, such as rotational core collapse, are particularly amenable to numerical investigations, as present-day computational resources allow for more accurate calculations than in full three dimensions. The early phase of the merging of two compact objects, when the two bodies start to plunge from the innermost stable circular orbit, is a three-dimensional situation, although after the merging itself the scenario is again suitable for investigations in axisymmetry. On the other hand, Newtonian gravity does not correctly describe the dynamics of these processes, and one has to use general relativity for a proper description, as well as to compute waveforms, or at least some approximation better than the Newtonian one. Post-Newtonian approximations of the metric in the near zone match the features described before quite well, and probably most of these scenarios can still be handled without fully general relativistic calculations. Let $d$ be the typical dimension of the source, $v$ the typical velocity of the system, and $t$ the typical 
timescale of variation of the gravitational field; hence, the typical frequency of the gravitational waves in the wave zone is $1 / t$, and the wavelength is $\lambda \sim c t$, where $c$ is the speed of light. From the virial theorem it follows that $t$ is also the typical dynamical timescale, hence $d \sim v t$ and $v / c \sim d / c t \sim d / \lambda$. Therefore, a post-Newtonian expansion around $v / c \rightarrow 0$ is equivalent to an expansion around $d / \lambda \rightarrow 0$. For the typical scales involved in core collapse and neutron star evolution we get $d^{2} / \lambda^{2} \sim 0.05$. That estimate yields first and second order post-Newtonian corrections of about $5 \%$ and $0.3 \%$, respectively. We note, however, that although these corrections may seem to be small, the nonlinearity of the equations makes it difficult to predict their effects beforehand in a numerical simulation.

During the last two decades, calculations of gravitational waveforms from core collapse were done using different approaches for gravity (Müller 1982; Finn 1989; Mönchmeyer et al. 1991; Bonazzola \& Marck 1993; Yamada \& Sato 1995; Zwerger \& Müller 1997; Rampp et al. 1998; Dimmelmeier et al. 2001, 2002a,b; Fryer et al. 2002, 2004; Imamura et al. 2003; Kotake et al. 2003; Shibata \& Sekiguchi 2004a; Ott et al. 2004). First attempts were made in Newtonian gravity, using Eulerian codes with artificial viscosity (Müller 1982; Finn 1989; Mönchmeyer et al. 1991) to calculate the hydrodynamics. Pseudo-spectral methods were used by Bonazzola \& Marck (1993), who followed the collapse without including the bounce phase due to the appearance of numerical instabilities associated with the presence of a shock wave. The need for correctly treating the shock wave, which forms after core bounce, gradually led to the use of high-resolution shock-capturing (HRSC) schemes. Zwerger \& Müller (1997) first used HRSC methods to simulate a sequence of collapsing rotating polytropes in axisymmetry and provided a comprehensive waveform catalog. Extensions to $3 \mathrm{D}$ were carried out by Rampp et al. (1998). In both works a simple equation of state (Janka et al. 1993) was used. Recently there were attempts to include more realistic physics in Newtonian simulations: Ott et al. (2004) used the equation of state of Lattimer \& Swesty (1991), while Müller et al. (2004) also incorporated 2D neutrino transport and computed the gravitational wave emission produced by neutrino-driven convection.

A step forward to improve the treatment of the gravitational field was taken by Dimmelmeier et al. (2001, 2002a,b), who used the conformal flatness condition (CFC hereafter) of Isenberg, Wilson, and Mathews (see Isenberg 1978; Wilson et al. 1996). Note that in the spherical case CFC is not an approximation of general relativity but rather is exact. In a more general context it was shown that conformally flat gravity agrees with full general relativity at the level of the first post-Newtonian expansion (Kley \& Schäfer 1999). Therefore the CFC approximation reproduces quite well the main features of the models studied, i.e. quasi-spherical sources tractable by the post-Newtonian formalism. The major numerical advantage of the CFC approximation is that the $3+1$ metric equations are reduced to elliptic equations, which, in principle, makes their implementation more stable than in the standard formulations of the field equations commonly used in numerical relativity. Concerning the core collapse waveforms, Dimmelmeier et al. (2002b) found quantitative and also qualitative differences between Newtonian and CFC calculations. This demonstrates that a relativistic calculation of the spacetime dynamics is necessary for properly studying core collapse and the emitted gravitational waves. Recently this work in axisymmetry was extended to 3D by Dimmelmeier et al. (2005), who combined HRSC methods for the hydrodynamic equations and spectral methods for the metric equations.

First multidimensional core collapse simulations in full general relativity were reported by Siebel et al. (2003), who used the characteristic formulation of the Einstein equations. In these simulations the gravitational waveform extraction using the Bondi news function at future null infinity was hampered by gauge effects and numerical inaccuracies. Recently, Shibata \& Sekiguchi (2004a) performed simulations in axisymmetry using the so-called Cartoon method to solve the Einstein equations, finding remarkable agreement with the CFC results of Dimmelmeier et al. (2002b). Extensions of this work to full 3D are reported in Shibata \& Sekiguchi (2004b). Except for Siebel et al. (2003), all existing simulations thus far computed the gravitational wave signals using the Newtonian quadrupole formula.

In this paper we propose a new approximation scheme for the metric equations as an extension of the CFC approach. Our scheme consists of adding second post-Newtonian terms to the conformal flat metric. With the new approach, which we call $\mathrm{CFC}+$, the resulting metric equations are still elliptic, and they reduce to full general relativity in the spherical case. Therefore, we conserve the main advantages of the CFC approximation at small extra computational cost (since the additional elliptic equations are linear), while improving the computation of the dynamics and the metric. The aim of this paper is to compare the quality of the CFC+ approximation in different scenarios with that of the CFC approach. In particular we study two scenarios of current interest in relativistic astrophysics: the gravitational collapse of rotating stellar cores to neutron stars (the relevant mechanism responsible for core collapse supernovae of types $\mathrm{II} / \mathrm{Ib} / \mathrm{Ic}$ ) and the stability and oscillations of rotating neutron stars.

This paper is organized as follows. In Sect. 2 we present the mathematical framework and introduce the general relativistic equations for the hydrodynamics and the spacetime metric that we implement in the numerical code. Particular emphasis is given to the derivation of the $\mathrm{CFC}+$ equations (for technical details see Appendices A, B, and C). Section 3 is devoted to describing the numerical methods for solving the hydrodynamics and metric equations. We go on in this section to explain the procedure we follow to extract gravitational waves (see also Appendix D). In Sect. 4 we specify the models used for the simulations of rotating relativistic stars. The results from the simulations of evolutions of rotating neutron stars and rotational stellar core collapse to a neutron star are discussed in Sects. 5 and 6, respectively. Our conclusions are summarized in Sect. 7.

We use a spacelike signature $(-,+,+,+)$ and units in which $c=G=1$, except in some passages, particularly in the Appendices, where $c$ and $G$ are retained for a better understanding of the post-Newtonian expansion. Greek indices run from 0 to 3, Latin indices from 1 to 3, and we adopt the standard Einstein convention for summation over repeated indices. 
The indices of 3-vectors and 3-tensors are raised and lowered by means of the 3-metric.

\section{Mathematical framework}

We adopt the $3+1$ formalism (Arnowitt et al. 1962) to foliate spacetime with a metric $g_{\mu \nu}$ into a set of non-intersecting spacelike hypersurfaces. The line element reads

$\mathrm{d} s^{2}=-\alpha^{2} \mathrm{~d} t^{2}+\gamma_{i j}\left(\mathrm{~d} x^{i}+\beta^{i} \mathrm{~d} t\right)\left(\mathrm{d} x^{j}+\beta^{j} \mathrm{~d} t\right)$,

with $\alpha$ being the lapse function, which describes the rate of advance of time along a timelike unit vector $n^{\mu}$ normal to a hypersurface, $\beta^{i}$ being the spacelike shift 3 -vector, which describes the motion of coordinates within a surface, and $\gamma_{i j}$ being the spatial 3-metric.

\subsection{Hydrodynamic equations}

The hydrodynamic evolution of a relativistic perfect fluid with rest-mass current $J^{\mu}=\rho u^{\mu}$ and energy-momentum tensor $T^{\mu \nu}=\rho h u^{\mu} u^{v}+P g^{\mu \nu}$ in a (dynamic) spacetime is determined by a system of local conservation equations, which read

$\nabla_{\mu} J^{\mu}=0, \quad \nabla_{\mu} T^{\mu v}=0$,

where the covariant derivative is denoted by $\nabla_{\mu}$. The fluid is specified by its rest mass density $\rho$, its specific enthalpy $h=$ $1+\epsilon+P / \rho$, its pressure $P$, its specific internal energy $\epsilon$, and its 4 -velocity $u^{\mu}$. The pressure is determined by an equation of state (EOS) $P=P(\rho, \epsilon)$.

The 3-velocity of the fluid, as measured by an Eulerian observer at rest in a spacelike hypersurface $\Sigma_{t}$, is

$v^{i}=\frac{u^{i}}{\alpha u^{t}}+\frac{\beta^{i}}{\alpha}$.

Following the work of Banyuls et al. (1997) we introduce a set of variables in terms of the primitive (physical) hydrodynamic variables $\left(\rho, v^{i}, \epsilon\right)$, whose associated densities are conserved:

$D=\rho W$,

$S^{i}=\rho h W^{2} v^{i}$,

$\tau=\rho h W^{2}-P-D$.

In the above expressions, $W$ is the Lorentz factor defined as $W=\alpha u^{t}$, which satisfies the relation $W=1 / \sqrt{1-v_{i} v^{i}}$.

The previous choice allows us to write the conservation laws (2) as a first-order, flux-conservative hyperbolic system of equations (Banyuls et al. 1997):

$\frac{1}{\sqrt{-g}}\left[\frac{\partial \sqrt{\gamma} \boldsymbol{U}}{\partial t}+\frac{\partial \sqrt{-g} \boldsymbol{F}^{i}}{\partial x^{i}}\right]=\boldsymbol{Q}$,

with the state vector, flux vector, and source vector given by

$\boldsymbol{U}=\left[D, S_{j}, \tau\right]$,

$\boldsymbol{F}^{i}=\left[D \hat{v}^{i}, S_{j} \hat{v}^{i}+\delta_{j}^{i} P, \tau \hat{v}^{i}+P v^{i}\right]$,

$\boldsymbol{Q}=\left[0, \frac{1}{2} T^{\mu \nu} \frac{\partial g_{\mu \nu}}{\partial x^{j}}, \alpha\left(T^{\mu 0} \frac{\partial \ln \alpha}{\partial x^{\mu}}-T^{\mu v} \Gamma_{\mu \nu}^{0}\right)\right]$.
Here $\hat{v}^{i}=v^{i}-\beta^{i} / \alpha$, and $\sqrt{-g}=\alpha \sqrt{\gamma}$, with $g=\operatorname{det}\left(g_{\mu \nu}\right)$ and $\gamma=\operatorname{det}\left(\gamma_{i j}\right)$ being the determinant of the 4-metric and 3-metric, respectively, while $\Gamma_{\mu \nu}^{\lambda}$ are the Christoffel symbols.

For convenience, we also define the following modified conserved quantities:

$D^{*}=\sqrt{\bar{\gamma}} D, \quad S_{i}^{*}=\sqrt{\bar{\gamma}} S_{i}$.

The determinant $\bar{\gamma}$ is actually the ratio of $\gamma$ evaluated on some coordinate grid $\left\{x^{\mu}\right\}$ and the determinant $\hat{\gamma}$ of the flat metric $\hat{\gamma}_{i j}$ on the same grid: $\bar{\gamma}=\gamma / \hat{\gamma}$.

\section{2. $3+1$ metric equations}

In the $3+1$ formalism, the Einstein equations split into (i) evolution equations for the 3-metric $\gamma_{i j}$ and the extrinsic curvature $K_{i j}$,

$$
\begin{aligned}
\partial_{t} \gamma_{i j} & \left.=-2 \alpha K_{i j}+2 \nabla_{(i} \beta_{j}\right), \\
\partial_{t} K_{i j} & =-\nabla_{i} \nabla_{j} \alpha+\alpha\left(R_{i j}+K K_{i j}-2 K_{i k} K_{j}^{k}\right) \\
& +\beta^{k} \nabla_{k} K_{i j}+2 K_{k(i} \nabla_{j)} \beta^{k}-8 \pi \alpha\left(S_{i j}-\frac{\gamma_{i j}}{2}\left(S_{k}^{k}-E\right)\right),
\end{aligned}
$$

and (ii) constraint equations,

$R+K^{2}-K_{i j} K^{i j}-16 \pi E=0$,

$\nabla_{i}\left(K^{i j}-\gamma^{i j} K\right)-8 \pi S^{j}=0$,

which must be fulfilled at every spacelike hypersurface.

In these equations $\nabla_{i}$ is the covariant derivative with respect to the 3-metric $\gamma_{i j}, K_{i j}$ the extrinsic curvature, $R_{i j}$ the Ricci tensor on $\Sigma_{t}, R$ the scalar curvature and $K=K_{i}^{i}$ the trace of the extrinsic curvature. Brackets around indices indicate symmetrization. The projection of the energy-momentum tensor onto the spatial hypersurface is defined as $S_{i j}=\rho h W^{2} v_{i} v_{j}+\gamma_{i j} P$, while $E=\rho h W^{2}-P$ is the total energy per volume unit as measured by an observer at rest in $\Sigma_{t}$.

The ADM gauge, which we will be using in the derivation of the metric equations in Sects. 2.3 and 2.4, is defined as that gauge for which the 3-metric can be decomposed into a conformally flat term plus a transverse traceless term,

$\gamma_{i j}=\phi^{4} \hat{\gamma}_{i j}+h_{i j}^{\mathrm{TT}}$

with

$h_{i j}^{\mathrm{TT}} \hat{\gamma}^{i j}=0, \quad \hat{\gamma}^{i k} \hat{\nabla}_{k} h_{i j}^{\mathrm{TT}}=0$,

where $\hat{\gamma}_{i j}$ is the flat metric (with inverse $\hat{\gamma}^{i j} ; \hat{\gamma}_{i j}=\hat{\gamma}^{i j}=\delta_{i j}$ in Cartesian coordinates), $\phi$ the conformal factor, and $h_{i j}^{\mathrm{TT}}$ is transverse and traceless. The conjugate momentum $\pi^{i j}$ of $\gamma_{i j}$ is traceless as well:

$\pi^{i j} \hat{\gamma}_{i j}=0$

\subsection{CFC metric equations}

In spherical symmetry $h_{i j}^{\mathrm{TT}}=0$, i.e. the 3 -metric is conformally flat $(\mathrm{CF})$. A first reasonable approximation in quasi-spherical scenarios consists of imposing a vanishing $h_{i j}^{\mathrm{TT}}$ in Eq. (16):

$\gamma_{i j}=\phi^{4} \hat{\gamma}_{i j}$ 
This is the conformal flatness condition or Isenberg-Wilson-Mathews approximation (Isenberg 1978; Wilson et al. 1996). In the ADM gauge a conformally flat 3 -metric ensures the maximal slicing condition, $K=0$. It can hence be shown that the $3+1$ metric Eqs. (12)-(15) reduce to a set of five coupled elliptic (Poisson-like) equations for the metric components,

$$
\begin{aligned}
& \hat{\Delta} \phi=-2 \pi \phi^{5}\left(\rho h W^{2}-P+\frac{K_{i j} K^{i j}}{16 \pi}\right), \\
& \hat{\Delta}(\alpha \phi)=2 \pi \alpha \phi^{5}\left(\rho h\left(3 W^{2}-2\right)+5 P+\frac{7 K_{i j} K^{i j}}{16 \pi}\right), \\
& \hat{\Delta} \beta^{i}=16 \pi \alpha \phi^{4} S^{i}+2 \phi^{10} K^{i j} \hat{\nabla}_{j}\left(\frac{\alpha}{\phi^{6}}\right)-\frac{1}{3} \hat{\nabla}^{i} \hat{\nabla}_{k} \beta^{k},
\end{aligned}
$$

where $\hat{\nabla}$ and $\hat{\Delta}=\hat{\gamma}^{i j} \hat{\nabla}_{i} \hat{\nabla}_{j}$ are the flat space Nabla and Laplace operators, respectively.

Applying the CFC to the traceless part of Eq. (12) yields the following relation between the conformal factor and the components of the shift vector:

$\partial_{t} \phi=\frac{\phi}{6} \nabla_{k} \beta^{k}$

With this, Eq. (12) transforms into an expression for the extrinsic curvature components:

$K_{i j}=\frac{1}{2 \alpha}\left(\nabla_{i} \beta_{j}+\nabla_{j} \beta_{i}-\frac{2}{3} \gamma_{i j} \nabla_{k} \beta^{k}\right)$.

As a consequence of setting the off-diagonal elements of $\gamma_{i j}$ to zero, the degrees of freedom representing gravitational waves are removed from the spacetime. Therefore, we calculate gravitational waveforms in a post-processing step using the quadrupole formula. Note that at that approximation level the ADM gauge is equivalent to the maximal slicing quasiisotropic (MSQI) gauge, i.e. maximal slicing $(K=0)$ plus quasi-isotropic coordinates $\left(g_{r \theta}=0\right.$ and $g_{\theta \theta}=g_{r r}$ in spherical coordinates in an orthonormal basis).

\section{4. $C F C+$ metric equations}

We improve the metric approximation by adding to the CFC metric the second post-Newtonian deviation from isotropy. We call this approximation $\mathrm{CFC}+$. Compared to the $\mathrm{CFC}$ metric Eq. (19) the $\mathrm{CFC}+$ metric

$\gamma_{i j}=\phi^{4} \hat{\gamma}_{i j}+h_{i j}^{2 \mathrm{PN}}$

now includes a new traceless and transverse term $h_{i j}^{2 \mathrm{PN}}$, which is identical to $h_{i j}^{\mathrm{TT}}$ in the decomposition (16) up to the second post-Newtonian expansion. As described in Appendix A this higher order correction $h_{i j}^{2 \mathrm{PN}}$ is the solution of the tensor Poisson equation (Schäfer 1990)

$\hat{\Delta} h_{i j}^{2 \mathrm{PN}}=\hat{\gamma}_{i j}^{\mathrm{TT} k l} F_{k l}$,

where the source $F_{k l}$ is given by

$F_{k l}=-4 \hat{\nabla}_{k} U \hat{\nabla}_{l} U-16 \pi \frac{S_{k}^{*} S_{l}^{*}}{D^{*}}$, with $U$ being the "Newtonian" potential, which is the solution of the Poisson equation

$\hat{\Delta} U=-4 \pi D^{*}$.

The operator $\hat{\gamma}_{i j}^{\mathrm{TT} k l}$ is the transverse, trace-free (TT) projector as defined in Eq. (A.6).

Of the original CFC metric Eqs. (20)-(22) for $\phi, \alpha$, and $\beta^{i}$, only the equation for the lapse function, Eq. (21), has to be modified. It now explicitly depends on the second postNewtonian correction $h_{i j}^{2 \mathrm{PN}}$ :

$\hat{\Delta}(\alpha \phi)=[\hat{\Delta}(\alpha \phi)]_{h_{i j}^{2 \mathrm{PN}}=0}-\hat{\gamma}^{i k} \hat{\gamma}^{j l} h_{i j}^{2 \mathrm{PN}} \hat{\nabla}_{k} \hat{\nabla}_{l} U$

The metric equations for the conformal factor $\phi$ and the shift vector $\beta^{i}$ remain unaltered. However, the $\mathrm{CFC}+$ corrections couple implicitly, as the source terms on their right-hand sides depend on $\alpha$.

The computation of $h_{i j}^{2 \mathrm{PN}}$, i.e. the inversion of the tensor Poisson Eq. (26) by means of the Poisson integral operator $\hat{\Delta}^{-1}$, is simplified by the introduction of intermediate potentials $\mathcal{S}, \mathcal{S}_{i}, \mathcal{T}_{i}, \mathcal{R}_{i}$, and $\mathcal{S}_{i j}$, which are solutions of the following scalar/vector/tensor-Poisson equations:

$\hat{\Delta} \mathcal{S}=-4 \pi \frac{S_{i}^{*} S_{j}^{*}}{D^{*}} x^{i} x^{j}$,

$\hat{\Delta} \mathcal{S}_{i}=\left[-4 \pi \frac{S_{i}^{*} S_{j}^{*}}{D^{*}}-\hat{\nabla}_{i} U \hat{\nabla}_{j} U\right] x^{j}$,

$\hat{\Delta} \mathcal{T}_{i}=\left[-4 \pi \frac{S_{j}^{*} S_{k}^{*}}{D^{*}}-\hat{\nabla}_{j} U \hat{\nabla}_{k} U\right] \hat{\gamma}^{j k} \hat{\gamma}_{i l} x^{l}$,

$\hat{\Delta} \mathcal{R}_{i}=\hat{\nabla}_{i}\left(\hat{\nabla}_{j} U \hat{\nabla}_{k} U x^{j} x^{k}\right)$,

$\hat{\Delta} \mathcal{S}_{i j}=-4 \pi \frac{S_{i}^{*} S_{j}^{*}}{D^{*}}-\hat{\nabla}_{i} U \hat{\nabla}_{j} U$.

These equations are designed in such a way that their source terms approach zero like $r^{-3}$ when $r=|x|$ tends towards infinity, which ensures the existence of the corresponding Poisson integrals. With the help of these potentials, $h_{i j}^{2 \mathrm{PN}}$ can be simply expressed as

$$
\begin{aligned}
h_{i j}^{2 \mathrm{PN}}= & \hat{\Delta}^{-1}\left(\hat{\gamma}_{i j}^{\mathrm{TT} k l} F_{k l}\right) \\
=\frac{1}{2} & \mathcal{S}_{i j}-3 x^{k} \hat{\nabla}_{(i} \mathcal{S}_{j) k}+\frac{5}{4} \hat{\gamma}_{j m} x^{m} \hat{\nabla}_{i}\left(\hat{\gamma}^{k l} \mathcal{S}_{k l}\right) \\
& +\frac{1}{4} x^{k} x^{l} \hat{\nabla}_{i} \hat{\nabla}_{j} \mathcal{S}_{k l}+3 \hat{\nabla}_{(i} \mathcal{S}_{j)}-\frac{1}{2} x^{k} \hat{\nabla}_{i} \hat{\nabla}_{j} \mathcal{S}_{k} \\
& +\frac{1}{4} \hat{\nabla}_{i} \hat{\nabla}_{j} \mathcal{S}-\frac{5}{4} \hat{\nabla}_{i} \mathcal{T}_{j}-\frac{1}{4} \hat{\nabla}_{i} \mathcal{R}_{j} \\
& +\hat{\gamma}_{i j}\left[\frac{1}{4} \hat{\gamma}^{k l} \mathcal{S}_{k l}+x^{k} \hat{\gamma}^{l m} \hat{\nabla}_{m} \mathcal{S}_{k l}-\hat{\gamma}^{k l} \hat{\nabla}_{k} \mathcal{S}_{l}\right],
\end{aligned}
$$

as shown in Appendix B.

Due to the specific type of elliptic solvers employed in our computer code (see Sect. 3.2 below), it is not possible to use the inverse image method to evaluate the intermediate potentials up to spatial infinity. We instead solve Eqs. (30)-(34) assuming specific boundary conditions. These are determined from the 
multipole expansion $\mathcal{M}$ of the intermediate potentials $\mathcal{S}, \mathcal{S}_{i}$, $\mathcal{T}_{i}, \mathcal{R}_{i}$, and $\mathcal{S}_{i j}$. As detailed in Appendix C, these multipole expansions are given by

$$
\begin{aligned}
\mathcal{M}(\mathcal{S})= & \frac{1}{r} \int \mathrm{d}^{3} \boldsymbol{x} \sqrt{\hat{\gamma}}\left(\frac{S_{k}^{*} S_{l}^{*}}{D^{*}} x^{k} x^{\prime}\right), \\
\mathcal{M}\left(\mathcal{S}_{i}\right)= & \frac{1}{r} \int \mathrm{d}^{3} \boldsymbol{x} \sqrt{\hat{\gamma}} D^{*}\left(\frac{S_{i}^{*} S_{k}^{*}}{D^{* 2}} x^{k}+\hat{\gamma}_{i j} x^{j}\left(U+x^{k} \hat{\nabla}_{k} U\right)\right) \\
& +\frac{M^{2}}{2 r} \hat{\gamma}_{i j} n^{j}, \\
\mathcal{M}\left(\mathcal{T}_{i}\right)= & \frac{1}{r} \int \mathrm{d}^{3} \boldsymbol{x} \sqrt{\hat{\gamma}} D^{*}\left(\frac{\hat{\gamma}^{k l} S_{k}^{*} S_{l}^{*}}{D^{* 2}}+U\right)_{\gamma_{i j} x^{j}} \\
& +\frac{M^{2}}{2 r} \hat{\gamma}_{i j} n^{j}, \\
\mathcal{M}\left(\mathcal{R}_{i}\right)= & \frac{M^{2} \hat{\gamma}_{i j} n^{j}}{r}, \\
\mathcal{M}\left(\mathcal{S}_{i j}\right)= & \frac{1}{r} \int \mathrm{d}^{3} \boldsymbol{x} \sqrt{\hat{\gamma}} D^{*}\left(\frac{S_{i}^{*} S_{j}^{*}}{D^{* 2}}+\frac{1}{2} \hat{\gamma}_{i j} U+\hat{\gamma}_{i k} x^{k} \partial_{j} U\right),
\end{aligned}
$$

modulo $O\left(1 / r^{2}\right)$ corrections. $M=\int \mathrm{d}^{3} \boldsymbol{x} \sqrt{\hat{\gamma}} D^{*}$ is the baryonic rest mass. By evaluating the above expansions at a finite radius outside the star we obtain the necessary boundary conditions for $\mathcal{S}, \mathcal{S}_{i}, \mathcal{T}_{i}, \mathcal{R}_{i}$, and $\mathcal{S}_{i j}$.

\section{Numerical implementation}

The code used for the simulations presented in this paper is an extension of the one described in Dimmelmeier et al. (2002a,b), incorporating the new $\mathrm{CFC}+$ metric equations. The interested reader should refer to those articles for details beyond those discussed below.

We use spherical polar coordinates $\{t, r, \theta, \varphi\}$ and assume axial symmetry with respect to the rotation axis and symmetry with respect to the equatorial plane at $\theta=\pi / 2$. The computational grid is composed of $n_{r}$ radial zones and $n_{\theta}$ equidistant angular zones, whose specific values for the simulations reported here are given below. For convenience the radial cell-spacing is chosen equidistant for evolutions of equilibrium neutron stars and logarithmically spaced when simulating core collapse. As in the original code (Dimmelmeier et al. 2002a) the part of the grid outside the star is filled with an artificial atmosphere (as customary in finite difference codes similar to ours; see Font et al. 2002; Duez et al. 2002; Baiotti et al. 2004). This atmosphere obeys a polytropic equation of state and has a very low density such that its presence does not affect the dynamics of the star (see Dimmelmeier et al. 2002a, for details). Moreover, an extended grid containing no matter is used beyond the atmosphere for the CFC+ metric calculations, namely to properly capture the radial fall-off behavior of the metric potentials and to extract the gravitational radiation using components of $h_{i j}^{\mathrm{TT}}$ in the wave zone (see Sect. 6.2).

\subsection{Hydrodynamics solver}

The hydrodynamics solver performs the numerical integration of the system of hydrodynamic conservation Eqs. (7) using a high-resolution shock-capturing (HRSC) scheme. This method ensures numerical conservation of physically conserved quantities and a correct treatment of discontinuities such as shocks. In HRSC methods a Riemann problem has to be solved at each cell interface, which requires the reconstruction of the primitive variables $\left(\rho, v^{i}, \epsilon\right)$ at these interfaces. We use the PPM method of Colella \& Woodward (1984) for the reconstruction, which yields third order accuracy in space. The solution of the Riemann problems then provides the numerical fluxes at cell interfaces. To obtain this solution, the characteristic structure of the hydrodynamics equations is explicitely needed (Banyuls et al. 1997).

In contrast to CFC in the CFC+ approximation the metric does not have any zero off-diagonal elements, so we have to use the most general eigenvalues and eigenvectors in general relativity as reported in Font (2003). Once the spectral information is known, the numerical fluxes are computed by means of Marquina's approximate flux formula (Donat \& Marquina 1996). The time update of the conserved vector $\boldsymbol{U}$ is done using the method of lines in combination with a Runge-Kutta method with second order accuracy in time. Once the conserved quantities $\left(D, S_{i}, \tau\right)$ are updated in time, the primitive variables need to be recovered. This is done through an iterative Newton-Raphson method, as these variables cannot be obtained in closed form from the conserved variables.

We note that the sources $\boldsymbol{Q}$ of the hydrodynamic equations have been implemented in the code using a general form for the metric, although they can be simplified for a metric with vanishing nondiagonal terms in the 3-metric as, for example, in CFC.

\subsection{Metric solver}

The main feature of the approximations we are using for the metric is that only elliptic equations have to be solved to know the metric at each time step. In our approximate scheme we solve the equations hierarchically. First a solution of the Poisson Eq. (28) for $U$ is obtained. Then we solve the Eqs. (30)-(34) for the intermediate potentials $\mathcal{S}, \mathcal{S}_{i}, \mathcal{T}_{i}, \mathcal{R}_{i}$, and $\mathcal{S}_{i j}$, which we need to calculate $h_{i j}^{\mathrm{TT}}$ in Eq. (35). Finally, we use $h_{i j}^{\mathrm{TT}}$ to solve the modified equations for $\phi, \beta^{i}$, and $\alpha$, Eqs. (20), (22), (29). For each step we use different methods according to the mathematical characteristics of each equation.

The equation for the Newtonian potential $U$ is a linear Poisson equation with compact support sources, so standard methods for Poisson equations like integral solvers can be used to obtain a numerical solution. For an equation of the form

$\hat{\Delta} u(\boldsymbol{x})=f(\boldsymbol{x})$

the solution for the potential $u$ can be expanded in axisymmetry as

$$
\begin{aligned}
u(\boldsymbol{x}) & =-\frac{1}{4 \pi} \int_{0} \mathrm{~d}^{3} \boldsymbol{x}^{\prime} \sqrt{\hat{\gamma}} \frac{f(\boldsymbol{x})}{\left|\boldsymbol{x}-\boldsymbol{x}^{\prime}\right|} \\
& =-\frac{1}{2} \sum_{l=0}^{\infty} P_{l}(\mu)\left(u_{\mathrm{in}}^{(l)}(\boldsymbol{x})+u_{\mathrm{out}}^{(l)}(\boldsymbol{x})\right),
\end{aligned}
$$


where

$u_{\mathrm{in}}^{(l)}(\boldsymbol{x})=\frac{1}{r^{l+1}} \int_{\left|x^{\prime}\right|<R} \mathrm{~d} r^{\prime} \mathrm{d} \mu^{\prime} f\left(\boldsymbol{x}^{\prime}\right) r^{\prime l+2} P_{l}\left(\mu^{\prime}\right)$,

$u_{\text {out }}^{(l)}(\boldsymbol{x})=r^{l} \int_{\left|x^{\prime}\right|>R} \mathrm{~d} r^{\prime} \mathrm{d} \mu^{\prime} f\left(\boldsymbol{x}^{\prime}\right) \frac{1}{r^{\prime l-1}} P_{l}\left(\mu^{\prime}\right)$.

Here $P_{l}$ are the Legendre polynomials, and $\mu=\cos \theta$. We numerically integrate Eqs. (43), (44) by assuming $f\left(\boldsymbol{x}^{\prime}\right)$ to be constant inside each computational cell, integrating over $r^{\prime}$ and $\theta^{\prime}$ analytically within each cell, and then adding up the partial integrals to obtain the solution at every grid point of the computational domain. This method has been described and tested in Müller \& Steinmetz (1995) and Zwerger (1995), and successfully used by Zwerger \& Müller (1997) to calculate the Newtonian potential in axisymmetric core collapse simulations.

The Eqs. (30)-(34) for the intermediate potentials, which are needed to compute the second post-Newtonian corrections to CFC, can in general be solved separately as a scalar Poisson equation for $\mathcal{S}$, three vector Poisson equations for $\mathcal{S}_{i}$, $\mathcal{T}_{i}$, and $\mathcal{R}_{i}$, and a tensor Poisson equation for $\mathcal{S}_{i j}$. In the axisymmetric case we can take advantage of some additional simplifications: the $\varphi$-component of the vector-Poisson equations decouples from the $r$ - and $\theta$-components, and the $r \varphi$ - and $\theta \varphi$-components of the tensor-Poisson equation decouple from all other components, even though e.g. $\hat{\Delta} \mathcal{S}_{i} \neq(\hat{\Delta} \mathcal{S})_{i}$ (similar for the other vectors and tensor), which means that the various components in general couple to each other. Therefore, the equations can be grouped into 9 sets of linear elliptic equations: four sets of one equation, four sets of two equations, and one set of four equations, with coupling only within the respective set.

The discretization of each of the equations on the $\{r, \theta\}$-grid leads to 9 sparse linear matrix equations of the type

$\mathcal{A l} \boldsymbol{u}=\boldsymbol{f}$,

where $\boldsymbol{u}=u_{i j}^{k}$ is the vector of unknowns with $i, j$ labeling the grid points and $k$ ranging from 1 to 1,2 , or 4 depending on the number of coupled components. The vector of sources is respectively denoted as $\boldsymbol{f}=f_{i j}^{k}$, and $\mathcal{A}$ is a matrix which does not depend on $\boldsymbol{u}$, as the original system is linear. The linearity of the equations allows us to avoid an iteration scheme and to use instead the LU decomposition method to invert $\mathcal{A}$. The main advantage of the LU method is that the decomposition itself (which is the most time consuming step) only has to be done once at the beginning. Then it can be used to calculate the solution for different source vectors $f$ during the metric computations, which is computationally very fast and efficient. The LU decomposition is performed using standard LAPACK libraries for banded matrices. We use the monopole solution of Eqs. (36)-(40) as explicit outer boundary condition for the intermediate potentials. This procedure is only accurate far from the sources, and the matching of the metric to a monopole solution deteriorates if it is performed too close to a strongly gravitating nonspherical star. As a consequence when calculating the components of the spacetime metric we use a grid which extends well beyond both the boundary of the star and also the atmosphere, and apply the boundary condition at the outer boundary of this extended vacuum grid. An example of the influence of the location where the boundary condition is applied on the accuracy of the metric solution is presented in Sect. 5.1. Although the nonlinear metric equations for $\phi, \alpha$, and $\beta^{i}$ have source terms with noncompact support, the location of the outer grid boundary has much less influence on the accuracy of the numerical solution (see Dimmelmeier et al. 2005).

At this stage we have a numerical solution for $h_{i j}^{\mathrm{TT}}$ and are ready to solve the $\mathrm{CFC}+$ metric equations for the conformal factor $\phi$, the shift vector $\beta^{i}$, and the lapse function $\alpha$, Eqs. (20), (22), (29). For the comparison between CFC and CFC+ presented in Sects. 5 and 6, we also need to solve the CFC metric equations, which, we recall, are equivalent to the $\mathrm{CFC}+$ equations up to corrections in the metric equation for $\alpha$ (21). As both systems of equations are 5 nonlinear elliptic coupled Poissonlike equations, we can apply the same methods to solve them. In either case, we can write them in compact form as

$\hat{\Delta} \boldsymbol{u}(\boldsymbol{x})=\boldsymbol{f}(\boldsymbol{x} ; \boldsymbol{u}(\boldsymbol{x}))$,

where $\boldsymbol{u}=u^{k}=\left(\phi, \alpha \phi, \beta^{j}\right)$, and $\boldsymbol{f}=f^{k}$ is the vector of the respective sources. These five scalar equations for each component of $\boldsymbol{u}$ couple to each other via the source terms that in general depend on the various components of $\boldsymbol{u}$.

We use a fix-point iteration scheme in combination with the linear Poisson solver (42) described above to solve these equations. The value of $\boldsymbol{u}$ at each iteration $s$, denoted by $\boldsymbol{u}^{s}$, is set constant in the sources $\boldsymbol{f}$ to calculate a new value $\boldsymbol{u}^{s+1}$,

$\boldsymbol{u}^{s+1}(\boldsymbol{x})=\hat{\Delta}^{-1} \boldsymbol{f}\left(\boldsymbol{x} ; \boldsymbol{u}^{s}(\boldsymbol{x})\right)$

As a result the 5 previously coupled nonlinear equations reduce to a decoupled set of 5 linear scalar Poisson equations. The solution vector $\boldsymbol{u}^{s+1}$ at each iteration step is obtained by solving the associated 5 Poisson equations of the type (41) separately. After the computation of $\boldsymbol{u}^{s+1}$, the right-hand side of Eq. (47) is updated by replacing $\boldsymbol{u}^{s} \rightarrow \boldsymbol{u}^{s+1}$, which is used as a new starting value for the next iteration. Convergence to the desired numerical solution $\boldsymbol{u}$ is achieved when the relative variation $\left|\boldsymbol{u}^{s+1} / \boldsymbol{u}^{s}-1\right|$ of the numerical solution of $\boldsymbol{u}$ between two successive iterations is smaller than a certain threshold, which we set to $10^{-6}$. In the simulations reported here the metric solver successfully converges when using the flat metric as initial guess in each metric computation. However, to accelerate convergence, during the hydrodynamic evolution we take the metric from the previous metric computation as starting value for the subsequent one. Furthermore, we cut the Legendre polynomial expansion in Eq. (42) at $l=10$. For the CFC metric equations in axisymmetry the above method was recently discussed in detail in Dimmelmeier et al. (2005).

\subsection{Gravitational wave extraction}

For an isolated source of gravitational radiation the metric can be considered as asymptotically flat. In the wave zone, defined as a neighborhood of future radiative infinity $\mathcal{I}^{+}$for which $r \gg \lambda /(2 \pi)$, the metric can be decomposed into the Minkowski metric $\eta_{\mu \nu}$ plus a small linear perturbation $h_{\mu \nu}^{\text {rad }}$. By an appropriate choice of gauge it is always possible to make the latter 
quantity algebraically transverse and traceless, up to quadratic corrections. At the linear order we then have

$h_{i j}^{\mathrm{rad}}=P_{i j}^{\mathrm{TT} k l}\left(\gamma_{k l}-\hat{\gamma}_{k l}\right)$,

where $P_{i j}^{\mathrm{TT} k l}=\hat{\gamma}_{i(p} \hat{\gamma}_{q) j}\left[\left(n^{p} n^{k}-\hat{\gamma}^{p k}\right)\left(n^{q} n^{l}-\hat{\gamma}^{q l}\right)-\right.$ $\left(n^{p} n^{q}-\hat{\gamma}^{p q}\right)\left(n^{k} n^{l}-\hat{\gamma}^{k l}\right) / 2$ stands for the algebraic transverse traceless projector. We note that the above expression stays invariant with respect to asymptotically Minkowskian gauge transformations of the 3-metric. It involves only two independent components, $h_{+}$and $h_{\times}$, representing the two degrees of freedom of the gravitational waves.

At linear order in the wave zone, $h_{i j}^{\text {rad }}$ can be approximated by means of the Newtonian quadrupole formula in some Bondilike coordinate system. In this representation, the waveform depends only on the second time derivative (denoted by a double dot) of the mass quadrupole moment $Q_{i j}$ of the source,

$h_{i j}^{\mathrm{rad}}(\boldsymbol{x}, t) \approx h_{i j}^{\mathrm{quad}}(\boldsymbol{x}, t)=P_{i j}^{\mathrm{TT} k l} \frac{2 \ddot{Q}_{k l}\left(t_{\mathrm{ret}}\right)}{r}$,

with the retarded time $t_{\text {ret }}=t-r / c$. The argument of $\ddot{Q}_{k l}$ in the ADM gauge is not simply equal to $t-r / c$. It also contains a logarithmic correction (Schäfer 1990) $-2(M+$ $\left.O\left(1 / c^{2}\right)\right) G \ln [r /(c \eta)] / c^{3}+O\left(G^{2}\right)$, where $\eta$ denotes an arbitrary constant with the dimension of time that cancels a term entering the first tail contribution. For large distances near future null infinity $\mathcal{I}^{+}$, this correction cannot be neglected, but it may be omitted in investigations focusing on time dependences as it depends on $r$ alone. Following Finn (1989), the time derivatives in the standard quadrupole formula can be replaced by spatial derivatives making use of the equations of motion. This yields the so-called stress formula,

$\ddot{Q}_{i j}=2 \int \mathrm{d}^{3} \boldsymbol{x} \sqrt{\hat{\gamma}} D^{*}\left[\hat{\gamma}_{k\langle i} \hat{\gamma}_{j\rangle l} v^{k} v^{l}+x^{k} \hat{\gamma}_{k\langle i} \hat{\nabla}_{j\rangle} U\right]$,

where the brackets \langle\rangle denote the symmetric and trace-free sets of indices, e.g. $\hat{\gamma}_{k\langle i} \hat{\nabla}_{j\rangle} U=\left(\hat{\gamma}_{k i} \hat{\nabla}_{j} U+\hat{\gamma}_{k j} \hat{\nabla}_{i} U\right) / 2-\hat{\gamma}_{i j} \hat{\nabla}_{k} U / 3$.

In axisymmetry, $h_{i j}^{\text {rad }}$ has only one degree of freedom, $h_{+}$. In the quadrupole approximation and in spherical coordinates, it takes the form

$h_{+}^{\text {quad }}(x, t)=\frac{1}{8} \sqrt{\frac{15}{\pi}} \sin ^{2} \theta \frac{A_{20}^{\mathrm{E} 2}(t-r / c)}{r}$,

with $A_{20}^{\mathrm{E} 2} \propto \ddot{Q}_{\theta \theta}$ being the amplitude of the quadrupole signal. The above formula allows us to calculate waveforms directly from the sources with no need to know the dynamical part of the metric. It may be used with profit in the CFC approximation, for which the metric is isotropic and therefore has no gravitational wave content.

In the CFC+ approach, the non-conformal part of the 3-metric in the near zone at the dominant (second) postNewtonian order, $h_{i j}^{2 \mathrm{PN}}$, is the solution of the pseudo-Poisson Eq. (26), where the remainder $O\left(1 / c^{6}\right)$ has been removed. This equation has an elliptic character, so that the information it carries propagates instantaneously. As a result, $h_{i j}^{2 \mathrm{PN}}$ is neither wave-like nor associated to any retardation effects, reflecting the fact that the post-Newtonian expansion is only valid at distances comparable to the wavelength of the system $\lambda$. By contrast, we know that the full non-conformally flat part $h_{i j}^{\text {full }}$ of the 3-metric carries the dynamical degrees of freedom of the gravitational field. This indicates a relation between $h_{i j}^{\text {full }}$ and $h_{i j}^{\text {rad }}$ near $\mathcal{I}^{+}$(they are indeed equal). Now, remarkably, it turns out that there is also a link between $h_{i j}^{2 \mathrm{PN}}$ and the radiative metric. As explained in Appendix D, the algebraic transverse traceless projection of the former quantity evaluated at retarded time $t_{\text {ret }}$ behaves as $h_{i j}^{\text {quad }} / 8$ at future null infinity, $P_{i j}^{\mathrm{TT} k l} h_{k l}^{2 \mathrm{PN}}\left(\boldsymbol{x}, t_{\mathrm{ret}}\right) \sim h_{i j}^{\mathrm{quad}} / 8$, or in components,

$h_{+}^{2 \mathrm{PN}}\left(\boldsymbol{x}, t_{\mathrm{ret}}\right) \sim \frac{1}{8} h_{+}^{\mathrm{quad}}(\boldsymbol{x}, t)$,

$h_{\times}^{2 \mathrm{PN}}\left(\boldsymbol{x}, t_{\mathrm{ret}}\right) \sim \frac{1}{8} h_{\times}^{\text {quad }}(\boldsymbol{x}, t)$,

where $h_{+}^{2 \mathrm{PN}}$ can be trivially calculated from the combination $h_{+}=\left(h_{\theta \theta}^{\mathrm{TT}}-h_{\varphi \varphi}^{\mathrm{TT}}\right) / 2$, whereas $h_{\times}^{2 \mathrm{PN}}$ is equal to $h_{\theta \varphi}^{2 \mathrm{PN}}$.

We see that the two polarizations extracted directly from $h_{i j}^{2 \mathrm{PN}}$ in the wave zone of the true waves differ from the quadrupole waveforms by (i) a factor 8 and (ii) the absence of retardation. The gravitational waveforms can thus be evaluated directly from $h_{+}^{2 \mathrm{PN}}$ and $h_{\times}^{2 \mathrm{PN}}$ even though there are no propagating gravitational waves in the $\mathrm{CFC}+$ spacetime (which just means that there is no radiation back-reaction due to energy losses caused by gravitational waves). Furthermore, the wave amplitude can be deduced solely from $h_{+}^{2 \mathrm{PN}}$ in the axisymmetric case.

In our simulations we extract the waveforms at a distance $r \sim 50 \lambda /(2 \pi)$ in the equatorial plane. This ensures that we are indeed in the wave zone of the true waves where the Newtonian quadrupole formula applies. We checked that $h_{+}^{2 \mathrm{PN}} \propto \sin ^{2} \theta / r$ there, so that the gravitational wave amplitude $A_{20}^{\mathrm{E} 2}$ is approximately constant independent of the radius $r$ or angle $\theta$ (except near the rotation axis where $h_{+}^{2 \mathrm{PN}}$ vanishes). Due to the smallness of $h_{+}^{2 \mathrm{PN}}$ for $r \gg \lambda /(2 \pi)$, some numerical error appears because of the cancellations of the different terms in Eq. (35). That yields an offset in the gravitational wave signal that can be corrected as follows:

$h_{+}^{2 \mathrm{PN} \text { corrected }}=h_{+}^{2 \mathrm{PN}}-a \hat{\gamma}^{i j} h_{i j}^{2 \mathrm{PN}}$.

Although the term $\hat{\gamma}^{i j} h_{i j}^{2 \mathrm{PN}}$ should be zero in principle, it is numerically comparable to $h_{i j}^{2 \mathrm{PN}}$ in the wave zone; the parameter $a$ corrects the offset in the waveforms.

Notice that the link between $h_{i j}^{2 \mathrm{PN}}$ and $h_{i j}^{\mathrm{full}}$ is trivial in the near zone, where the post-Newtonian approximation is valid: $h_{i j}^{\text {full }} \approx h_{i j}^{2 \mathrm{PN}}$. We shall henceforth denote their common value as $h_{i j}^{\mathrm{TT}}$.

\section{Initial models}

As initial models for describing either rotating neutron stars or rotating stellar cores at the onset of gravitational collapse, we construct uniformly or differentially rotating relativistic polytropes in equilibrium. These are obtained using Hachisu's self-consistent field (HSCF) method as described in Komatsu et al. (1989a,b), which solves the general relativistic 
Table 1. Equatorial radius $r_{\mathrm{e}}$, axis ratio $r_{\mathrm{p}} / r_{\mathrm{e}}$, angular velocity $\Omega$, and ADM mass $M_{\mathrm{ADM}}$ for a sequence of uniformly rotating neutron stars used in this paper. Along the sequence the rotation rate increases from the spherical model RNS0 to the most rapidly rotating model RNS5, which rotates near the mass shedding limit at $\Omega_{\mathrm{K}}=5.363 \mathrm{kHz}$.

\begin{tabular}{ccccc}
\hline \hline Model & $r_{\mathrm{e}}[\mathrm{km}]$ & $r_{\mathrm{p}} / r_{\mathrm{e}}$ & $\Omega / \Omega_{\mathrm{K}}$ & $M_{\mathrm{ADM}}\left[M_{\odot}\right]$ \\
\hline RNS0 & 14.1 & 1.00 & 0.00 & 1.40 \\
RNS1 & 16.2 & 0.95 & 0.42 & 1.44 \\
RNS2 & 17.3 & 0.85 & 0.70 & 1.51 \\
RNS3 & 18.7 & 0.75 & 0.87 & 1.59 \\
RNS4 & 19.6 & 0.70 & 0.93 & 1.63 \\
RNS5 & 20.7 & 0.65 & 0.98 & 1.67 \\
\hline
\end{tabular}

hydrostatic equations for self-gravitating rotating matter distributions, whose pressure obeys the polytropic relation

$P=K \rho^{\gamma}$,

where $K$ is the polytropic constant and $\gamma$ the adiabatic index. The gauge used in the HSCF method is maximal slicing with quasi-isotropic coordinates (MSQI). The most general metric to describe these objects in the MSQI gauge is

$$
\begin{aligned}
\mathrm{d} s^{2}= & -\mathrm{e}^{2 \tilde{v}} \mathrm{~d} \tilde{t}^{2}+\mathrm{e}^{2 \tilde{\alpha}}\left(\mathrm{d} \tilde{r}^{2}+\tilde{r}^{2} \mathrm{~d} \tilde{\theta}^{2}\right) \\
& +\mathrm{e}^{2 \tilde{\beta}} \tilde{r}^{2} \sin \tilde{\theta}^{2}(\mathrm{~d} \tilde{\varphi}-\tilde{\omega} \mathrm{d} \tilde{t})^{2},
\end{aligned}
$$

where $\{\tilde{t}, \tilde{r}, \tilde{\theta}, \tilde{\varphi}\}$ are the coordinates in the MSQI gauge, and $\tilde{v}$, $\tilde{\alpha}, \tilde{\beta}$, and $\tilde{\omega}$ are metric potentials. Hereafter quantities with a tilde are in the MSQI gauge, and all other quantities are in the ADM gauge. The hydrostatic equilibrium equations can be numerically integrated by prescribing a value for the central density $\rho_{\mathrm{c}}$ and the rotation rate (selected by setting a ratio of polar radius $r_{\mathrm{p}}$ to equatorial radius $r_{\mathrm{e}}$ ), and by choosing a rotation law. As is common in the literature we use

$\Omega=\Omega_{\mathrm{c}} \frac{A^{2}}{A^{2}+d^{2}}$

as rotation law, where $\Omega$ is the angular velocity of the fluid as measured from infinity, $\Omega_{\mathrm{c}}$ its value at the center, and $d=$ $r \sin \theta$ the distance to the rotation axis. The positive constant $A$ parametrizes the rate of differential rotation, with $A \rightarrow \infty$ for a rigid rotator and $A \lesssim r_{\mathrm{e}}$ for differentially rotating stars.

For the study of rotating neutron stars, we choose the polytropic EOS (55) with $K=1.456 \times 10^{5}$ (in cgs units) and $\gamma=2$. We construct a sequence of uniformly rotating models with a central density $\rho_{\mathrm{c}}=7.95 \times 10^{14} \mathrm{~g} \mathrm{~cm}^{-3}$, and a ratio $r_{\mathrm{p}} / r_{\mathrm{e}}$ of polar to equatorial coordinate radius ranging from 1.00 (spherical model; labeled RNS0) to 0.65 (rapidly rotating model near the mass-shedding limit; labeled RNS5). A summary of important quantities specifying these models is given in Table 1. Note also that models with this choice of parameters are of widespread use in the literature (see e.g. Font et al. 2002; or Dimmelmeier et al. 2005, and references therein) and will be used in this work for comparison with previous results obtained with independent codes.

To model a stellar iron core as initial model for simulating rotational stellar core collapse to a neutron star we again utilize the HSCF method with the EOS parameters $K=4.897 \times 10^{14}$
Table 2. Parameters used in the core collapse simulations. The initial models are differentially rotating stellar cores specified by the parameter $A$ controling the degree of differential rotation (cf. Eq. (57)) and the ratio of rotational to potential energy, $\beta=T /|W|$. The collapse is initiated by reducing $\gamma$ from its initial value $4 / 3$ to $\gamma_{1}$. Additionally, the values for the initial equatorial radius $r_{\mathrm{e}}$ and the initial ADM mass $M_{\mathrm{ADM}}$ are given. The label $\mathrm{A} x \mathrm{~B} y \mathrm{G} z$ of each model is a combination of the initial rotation parameters $A$ and $\beta(\mathrm{A} x \mathrm{~B} y)$ and the value of $\gamma_{1}$ during collapse $(\mathrm{Gz})$.

\begin{tabular}{cccccc}
\hline \hline Model & $\begin{array}{c}A \\
{\left[10^{3} \mathrm{~km}\right]}\end{array}$ & $\begin{array}{c}\beta \\
{[\%]}\end{array}$ & $\begin{array}{c}r_{\mathrm{e}} \\
{[\mathrm{km}]}\end{array}$ & $\begin{array}{c}M_{\mathrm{ADM}} \\
{\left[M_{\odot}\right]}\end{array}$ & $\gamma_{1}$ \\
\hline A1B3G3 & 50.0 & 0.89 & 2247 & 1.46 & 1.31 \\
A1B3G5 & 50.0 & 0.89 & 2247 & 1.46 & 1.28 \\
A2B4G5 & 1.0 & 1.81 & 1739 & 1.50 & 1.28 \\
A4B5G5 & 0.5 & 4.03 & 1249 & 1.61 & 1.28 \\
\hline
\end{tabular}

(in cgs units) and $\gamma=4 / 3$, chosen to approximate the pressure profile in a degenerate electron gas. The initial central density is set to $\rho_{\text {c ini }}=10^{10} \mathrm{~g} \mathrm{~cm}^{-3}$. Again each initial model is further determined by its rotation profile parameter $A$ and the rotation rate, which is specified by the axis ratio $r_{\mathrm{p}} / r_{\mathrm{e}}$ or equivalently by the ratio of rotational energy and gravitational binding energy, $\beta=T /|W|$.

Following Dimmelmeier et al. (2002a) we use a hybrid EOS in the core collapse simulations. This EOS consists of a polytropic part $P_{\mathrm{p}}=K \rho^{\gamma}$ plus a thermal part $P_{\mathrm{th}}=\left(\gamma_{\mathrm{th}}-1\right) \epsilon_{\mathrm{th}}$, where $\gamma_{\text {th }}=1.5$ and $\epsilon_{\mathrm{th}}=\epsilon-\epsilon_{\mathrm{p}}$. The thermal contribution is chosen to take the rise of thermal energy due to shock heating into account. Details of this EOS can be found in Dimmelmeier et al. (2002a). Gravitational collapse is initiated by slightly decreasing $\gamma$ from its initial value to $\gamma_{1}<4 / 3$. As $\rho$ reaches nuclear density $\rho_{\text {nuc }}=2.0 \times 10^{14} \mathrm{~g} \mathrm{~cm}^{-3}$, we raise $\gamma$ to $\gamma_{2}=2.5$ and adjust $K$ accordingly to maintain monotonicity of the pressure. Due to this stiffening of the EOS the core undergoes a bounce. In Table 2 we present the main properties of models A1B3G3, A1B3G5, A2B4G5, and A4B5G5 used in this work to study core collapse. These models are identical to those with the same labels in the comprehensive core collapse study performed by Dimmelmeier et al. (2002b).

We note that when evolving the initial models constructed on the basis of the HSCF method (which uses the MSQI gauge) with the CFC+ evolution code (which uses the ADM gauge), we have to consider that in general the two gauges differ. This could potentially lead to an unsuitable matching of the data describing the initial models when evolved with the numerical code. Let us consider the most general metric in a generic dynamic scenario,

$\gamma_{i j}=\phi^{4} \hat{\gamma}_{i j}+h_{i j}^{\mathrm{TT}}(\mathrm{ADM}$ gauge $)$

$\tilde{\gamma}_{i j}=\tilde{\phi}^{4} \hat{\gamma}_{i j}+\tilde{\gamma}_{\langle i j\rangle}$ (MSQI gauge).

In general $\tilde{\gamma}_{\langle i j\rangle}$ is not transverse, so that the ADM gauge and the MSQI gauge differ. To quantify the differences between both gauges it is relevant to compare the traceless part of the 3-metric $\gamma_{\langle i j\rangle}$ and the trace $\hat{\gamma}^{i j} \gamma_{i j}=3 \phi^{4}$ itself. For an 


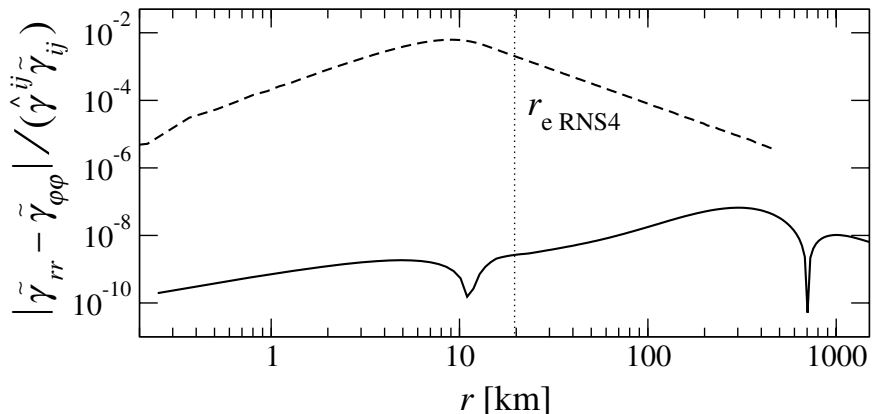

Fig. 1. Deviation from conformal flatness along the equatorial plane for a typical rotating stellar core initial model (model A1B3; solid line), and for a typical rotating neutron star in equilibrium with axis ratio $r_{\mathrm{p}} / r_{\mathrm{e}}=0.7$ (model RNS4; dashed line). The vertical dotted line indicates the equatorial stellar radius for the neutron star.

equilibrium stellar model constructed within the MSQI gauge one obtains

$\tilde{\gamma}_{\langle i j\rangle}=\left(\tilde{\gamma}_{r r}-\tilde{\gamma}_{\varphi \varphi}\right) \operatorname{diag}\left(\frac{1}{3}, \frac{1}{3},-\frac{2}{3}\right)$.

In Fig. 1 we plot equatorial profiles of $\left|\tilde{\gamma}_{r r}-\tilde{\gamma}_{\varphi \varphi}\right| /\left(\hat{\gamma}^{i j} \tilde{\gamma}_{i j}\right)$ for (i) the equilibrium model A1B3 used as initial data for core collapse simulations (lower curve; cf. Table 2), and for (ii) the equilibrium rotating neutron star model RNS4 (upper curve; cf. Table 1). In both cases, but especially in the (only weakly relativistic) collapse initial model, we observe that deviations from conformal flatness are negligible, which makes the initial models built with HSCF method suitable for time evolution in the CFC approximation. It also shows that the differences between both gauges are very small, namely of the same order of magnitude as $\tilde{\gamma}_{\langle i j\rangle}$. Consequently, the use of initial models computed in the MSQI gauge for evolutions using the ADM gauge is entirely justified since the differences are negligible and only appear at the second post-Newtonian order.

\section{Rotating neutron stars}

\subsection{Effects of the boundary on the metric solution}

As mentioned in Sect. 3.2 the location where the boundary conditions for the $\mathrm{CFC}+$ intermediate potentials are imposed can have a significant impact on the accuracy of the metric solution. Additionally, the extension of the numerical grid is also of paramount importance for numerically solving the CFC equations to obtain the conformal factor, the shift vector, and the lapse function. As the sources of these equations have noncompact support, it is necessary to integrate out far enough in radius to obtain the desired accuracy for the numerical solution.

To accomplish this we use an extended (vacuum) grid going far beyond the actual stellar boundary. Monopole behavior at the outer boundary of this vacuum domain has been checked by comparison with calculations done imposing zero values for the potentials as outer boundary conditions at extremely large distances (see Fig. 2). Convergence tests with different parameters for the outer grid (number of cells, distance of the outer boundary, amplification factor of the grid) were performed.

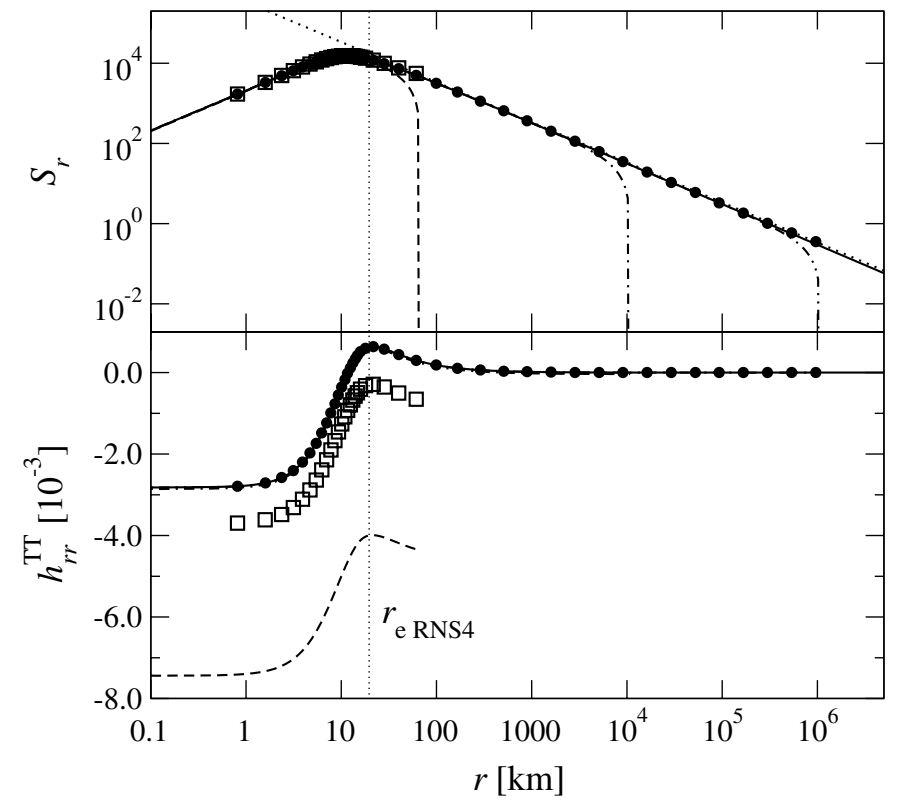

Fig. 2. Effects of the outer boundary conditions on the equatorial radial profile of the intermediate potential $\mathcal{S}_{r}$ (top panel) and the metric component $h_{r r}^{\mathrm{TT}}$ (bottom panel) for the rotating neutron star model RNS4. Zero-value boundary conditions are imposed at $62 \mathrm{~km}$ (dashed line), at $10^{4} \mathrm{~km}$ (dashed-dotted line), at $10^{6} \mathrm{~km}$ (dashed-dot-dotted line), and at $10^{11} \mathrm{~km}$ (solid line). Alternatively, monopole boundary conditions are imposed at $62 \mathrm{~km}$ (open boxes) and at $10^{6} \mathrm{~km}$ (filled circles). Overplotted to $\mathcal{S}_{r}$ is the monopole behavior (dotted line).

The conclusion of these tests is that for simulations of rotating neutron stars we need to use a numerical grid consisting of $n_{\theta}=30$ angular cells and $n_{r}=250$ radial cells (of which 100 are equally spaced inside the neutron star and 150 are logarithmically spaced for the atmosphere) to correctly capture the conformally flat part of the metric. In order to impose outer boundary conditions for the CFC+ metric potentials, an extra grid of 70 radial cells extending out to $10^{6} \mathrm{~km}$ needs to be added.

\subsection{CFC+ corrections to the 3-metric}

We now turn to measure the magnitude of the $\mathrm{CFC}+$ corrections to the metric of our sample of rotating neutron star models (cf. Table 1). First, from the distribution of the hydrodynamic variables in the equilibrium models provided by the HSCF method we recompute the $\mathrm{CFC}+$ metric components. For equilibrium configurations, i.e. in an axisymmetric stationary spacetime, the components $h_{r \varphi}^{\mathrm{TT}}$ and $h_{\theta \varphi}^{\mathrm{TT}}$ vanish. All other components are shown in Fig. 3 for different models with increasing rotation rate. Note that as discussed in Sect. 4 the ADM gauge is not quasi-isotropic in the CFC+ approach, because $h_{r \theta}^{\mathrm{TT}} \neq 0$ and $h_{r r}^{\mathrm{TT}} \neq h_{\theta \theta}^{\mathrm{TT}}$. Therefore, a direct comparison with the full general relativistic metric calculated in the MSQI gauge is not possible. As expected, $h_{i j}^{\mathrm{TT}}$ vanishes for the spherical case RNS0, in which both CFC and CFC+ agree exactly with general relativity, and their metrics have no traceless and transverse part. As we increase the rotation rate from RNS1 to RNS5, the value of $h_{i j}^{\mathrm{TT}}$ increases, resulting in a departure of 


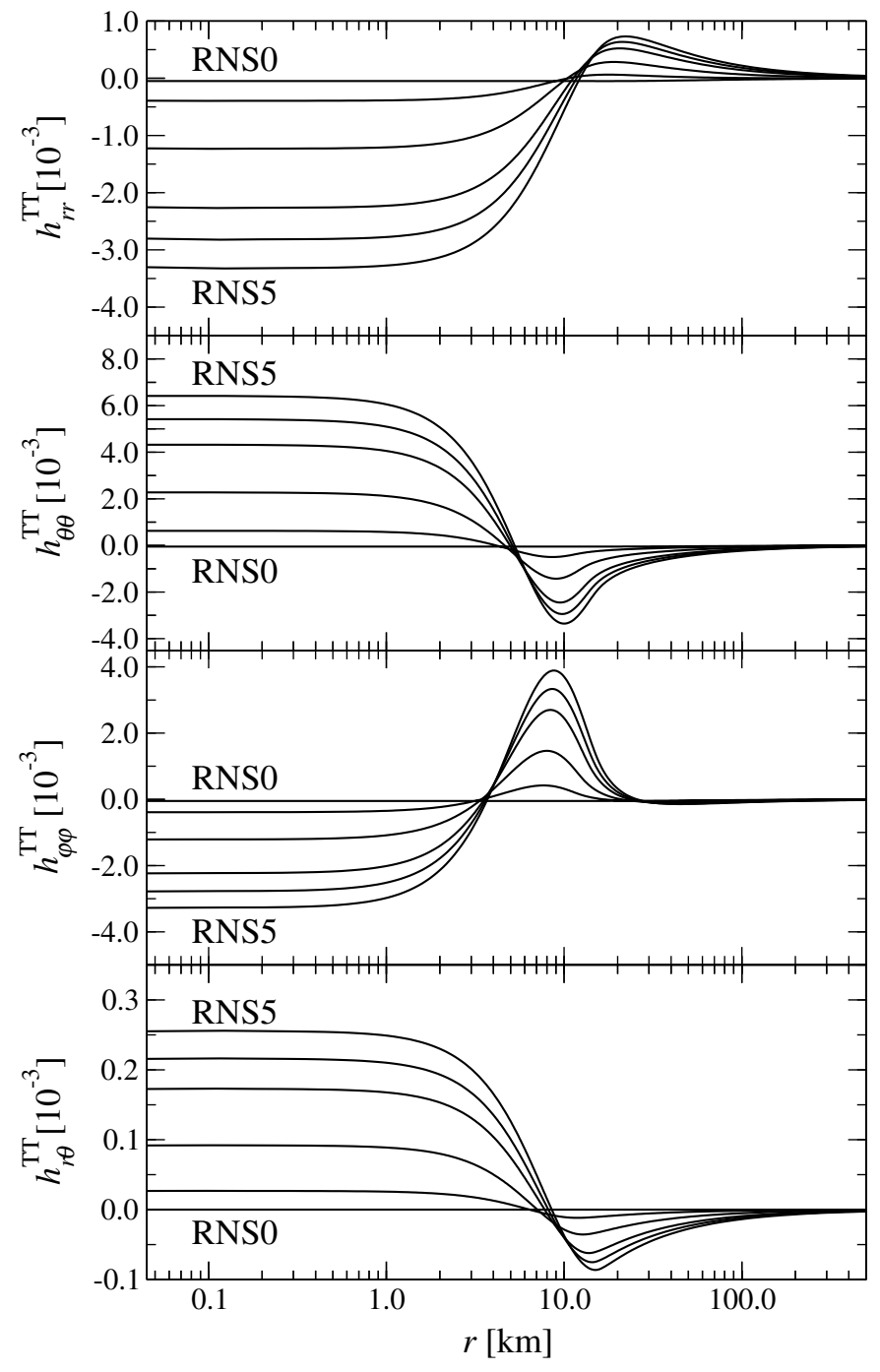

Fig. 3. Radial profiles at the equator of the non-vanishing components of $h_{i j}^{\mathrm{TT}}$ for the sequence of models RNS0 to RNS5. The strength of the correction $h_{i j}^{\mathrm{TT}}$ increases with the rotation rate. The equatorial radius of each model is given in Table 1 .

the metric from conformal flatness. As $h_{i j}^{\mathrm{TT}}$ is a correction to the conformally flat 3-metric (which is of order unity, $\phi \sim 1$ ), it can be shown that even in our most extreme case RNS5, which is very near to the mass shedding limit, the values of $h_{i j}^{\mathrm{TT}}$ amount to a correction of only about $1 \%$. Therefore, qualitative differences with respect to the CFC approximation are not expected in the dynamics of such objects.

\subsection{Oscillations of rotating neutron stars}

A further test of the new approximation for the metric is provided by studying pulsations of rotating neutron stars. To this aim we perturb the neutron star models described in Sect. 4 with a density perturbation of the form $\rho_{\text {pert }}=\rho[1+$ $\left.a \cos \left(\pi r /\left(2 r_{\mathrm{e}}\right)\right)\right]$, where $a$ is an arbitrary parameter controlling the strength of the perturbation. The perturbed models are evolved in time in two different ways, either considering coupled evolutions for the hydrodynamics and the metric, or evolving only the hydrodynamics in a fixed background metric
Table 3. Frequencies of small-amplitude quasi-radial pulsations for model RNS4. We compare the frequencies obtained from simulations with the present code (using either the CFC or the CFC+ approximation) with those obtained independently from a 3D full general relativistic code (GR). The results are extracted from time evolutions where the spacetime metric is kept fixed (Cowling approximation). The relative differences between the $\mathrm{CFC}+$ and the GR code are shown in the last column.

\begin{tabular}{ccccc}
\hline \hline Mode & $\begin{array}{c}f^{\text {CFC }} \\
{[\mathrm{kHz}]}\end{array}$ & $\begin{array}{c}f^{\mathrm{CFC}+} \\
{[\mathrm{kHz}]}\end{array}$ & $\begin{array}{c}f^{\mathrm{GR}} \\
{[\mathrm{kHz}]}\end{array}$ & $\begin{array}{c}\text { Rel. diff. } \\
{[\%]}\end{array}$ \\
\hline F & 2.48 & 2.48 & 2.468 & 0.5 \\
H1 & 4.39 & 4.39 & 4.344 & 1.1 \\
H2 & 6.30 & 6.30 & 6.250 & 0.8 \\
\hline
\end{tabular}

corresponding to the metric provided by the elliptic solvers at the first time step (an approximation commonly referred to in the literature as the Cowling approximation). Both metric approximations, $\mathrm{CFC}$ and $\mathrm{CFC}+$, are used to compare the respective results.

The oscillations of the stars can be observed in various hydrodynamic and metric quantities. In particular we monitor the central density $\rho_{\mathrm{c}}$, the radial velocity $v_{r}$ at half the stellar radius, and the gravitational wave amplitudes $A_{20}^{\mathrm{E} 2}$ extracted with the quadrupole formula. When Fourier transforming the time evolution of these quantities, distinctive peaks appear at the same (discrete) frequencies for any of these variables. Those frequencies can be identified with the quasi-normal modes of pulsation of the star, as described in Font et al. (2000). To further validate our approach we present the quasi-normal modes calculated in the CFC and CFC+ approximation in comparison with those reported by Font et al. (2002), which are calculated with a 3D code in full general relativity (without any approximation).

Table 3 shows the mode-frequencies for the fundamental mode (F), as well as for the first (H1) and second (H2) harmonics obtained in evolutions of model RNS4 in which the spacetime metric is kept fixed (Cowling approximation). The accuracy in the frequency values depends on the total time of the evolution, increasing as the evolution is extended. We evolved all models for $30 \mathrm{~ms}$, finding no significant deviations in the hydrodynamic profiles with respect to the original profiles of the equilibrium models. For such an evolution time the FFT yields a maximum frequency resolution of $0.03 \mathrm{kHz}$. Table 3 shows that no differences can be observed between the modefrequencies computed with $\mathrm{CFC}$ and $\mathrm{CFC}+$, and that, in addition, there is very good agreement with the general relativistic results, since the reported values are within the affordable resolution in frequency.

The corresponding results for the case of coupled evolutions of the spacetime metric and the hydrodynamics are shown in Tables 4 and 5 for the fundamental mode and the first harmonic, respectively. In these simulations, for the sake of computational efficiency and without affecting the dynamics, the metric is calculated every 100th hydrodynamic time step and extrapolated in between, as explained in Dimmelmeier et al. (2002a). As in the Cowling simulations, all models are evolved for $30 \mathrm{~ms}$. Even for the more rapidly rotating models, 
Table 4. Fundamental mode frequency $f_{\mathrm{F}}$ of small-amplitude quasiradial pulsations for a sequence of rotating polytropes with increasing ratio of polar to equatorial radius $r_{\mathrm{p}} / r_{\mathrm{e}}$. We compare the frequencies obtained from simulations with the present code using either the CFC or the CFC+ approximation with those obtained independently from a 3D full general relativistic code (GR). The results are extracted from coupled spacetime metric and hydrodynamic time evolutions. The relative differences between the CFC+ and the GR code are shown in the last column.

\begin{tabular}{lccccc}
\hline \hline Model & $r_{\mathrm{p}} / r_{\mathrm{e}}$ & $\begin{array}{c}f_{\mathrm{F}}^{\text {CFC }} \\
{[\mathrm{kHz}]}\end{array}$ & $\begin{array}{c}f_{\mathrm{F}}^{\text {CFC+ }} \\
{[\mathrm{kHz}]}\end{array}$ & $\begin{array}{c}f_{\mathrm{F}}^{\mathrm{GR}} \\
{[\mathrm{kHz}]}\end{array}$ & $\begin{array}{c}\text { Rel. diff. } \\
{[\%]}\end{array}$ \\
\hline RNS0 & 1.00 & 1.43 & 1.43 & 1.450 & 1.4 \\
RNS1 & 0.95 & 1.40 & 1.40 & 1.411 & 0.8 \\
RNS2 & 0.85 & 1.34 & 1.34 & 1.350 & 0.7 \\
RNS3 & 0.75 & 1.27 & 1.27 & 1.265 & 0.4 \\
RNS4 & 0.70 & 1.24 & 1.24 & 1.247 & 0.6 \\
RNS5 & 0.65 & 1.21 & 1.21 & 1.195 & 1.0 \\
\hline
\end{tabular}

Table 5. Same as Table 4 but for the frequency $f_{\mathrm{H} 1}$ of the first harmonic mode. In the model RNS5 this harmonic was not sufficiently excited by the perturbation chosen for a clear identification of its frequency.

\begin{tabular}{cccccc}
\hline \hline Model & $r_{\mathrm{p}} / r_{\mathrm{e}}$ & $\begin{array}{c}f_{\mathrm{H} 1}^{\mathrm{CFC}} \\
{[\mathrm{kHz}]}\end{array}$ & $\begin{array}{c}f_{\mathrm{H} 1}^{\mathrm{CF}+} \\
{[\mathrm{kHz}]}\end{array}$ & $\begin{array}{c}f_{\mathrm{H} 1}^{\mathrm{GR}} \\
{[\mathrm{kHz}]}\end{array}$ & $\begin{array}{c}\text { Rel. diff. } \\
{[\%]}\end{array}$ \\
\hline RNS0 & 1.00 & 3.97 & 3.97 & 3.958 & 0.3 \\
RNS1 & 0.95 & 3.87 & 3.87 & 3.852 & 0.5 \\
RNS2 & 0.85 & 3.95 & 3.95 & 3.867 & 2.0 \\
RNS3 & 0.75 & 3.98 & 3.98 & 4.031 & 1.3 \\
RNS4 & 0.70 & 4.02 & 4.02 & 3.887 & 2.0 \\
RNS5 & 0.65 & - & - & 3.717 & - \\
\hline
\end{tabular}

no differences in the frequencies from the $\mathrm{CFC}$ and $\mathrm{CFC}+$ simulations can be found. This result can again be explained by the smallness of the components of $h_{i j}^{\mathrm{TT}}$, which does not modify the dynamics considerably. Furthermore, the results agree to high precision with the GR results of Font et al. (2002) within the limits set by the temporal and spatial resolution.

We emphasize that, for accurately extracting the oscillation mode frequencies, the code has to maintain the initial equilibrium configuration in a hydrodynamical evolution for many rotation periods (usually several tens of periods). Independently of the approximation assumed for the metric (either CFC or $\mathrm{CFC}+$ ) and the (small) gauge mismatch, we tested that our code is able to perform that task successfully.

\section{Rotational core collapse}

We now present results of simulations of rotational core collapse to neutron stars. The core collapse models we have selected (see Table 2) are representative of the different types of collapse dynamics and gravitational radiation waveforms observed in the CFC simulations of Dimmelmeier et al. (2002a): A1B3G5 as type I (regular collapse), A2B4G5 as type II (multiple bounce collapse), A1B3G5 as type III (rapid collapse), and A4B5G5 as a case with extreme rotation, i.e. a strongly and highly differentially rotating core with an initial torus-like structure which is strongly enhanced during collapse. We use a numerical grid consisting of $n_{\theta}=30$ equally spaced angular cells and $n_{r}=300$ logarithmically spaced radial cells covering the star. In order to calculate $h_{i j}^{\mathrm{TT}}$ and extract waveforms, an extra grid of 300 radial cells extending out to $10^{11} \mathrm{~km}$ needs to be added (600 cells for model A2B4G1).

\subsection{Collapse dynamics}

In Figs. 4 and 5 we compare the time evolution of selected matter and metric quantities for all four collapse models considered using both the CFC and the CFC+ approximation. We show the time evolution of the central density $\rho_{\mathrm{c}}$, which is a representative quantity of the hydrodynamic evolution. We present $\rho_{\mathrm{c}}$ for all models except model A4B5G5 for which the time evolution of the maximum density $\rho_{\max }$ is used instead. In this model the density maximum is not attained at the center due to the strong differential rotation. The evolution of the central density in models A1B3G3 and A1B3G5 (see Fig. 4) shows a distinctive rise during collapse until $\rho_{\mathrm{c}}$ reaches its maximum at the time of bounce $t_{\mathrm{b}}$ (at $t_{\mathrm{b}} \sim 49 \mathrm{~ms}$ and $t_{\mathrm{b}} \sim 30 \mathrm{~ms}$, respectively). Later on, the density oscillates around the new equilibrium value of the compact remnant (which can be identified with the new-born proto-neutron star). These oscillations are highly damped due to the presence of an extended stellar envelope surrounding the proto-neutron star. Note that in models A2B4G1 and A4B5G5 (see Fig. 5), the collapse is stopped by rotation before nuclear matter density is reached, as strong centrifugal forces build up during the collapse. As a result, the evolution of model A2B4G1 is characterized by consecutive multiple bounces, while the centrifugal hang-up in model A4B5G5 causes a single bounce below nuclear matter density, leaving a low density proto-neutron star behind.

The top panels of Figs. 4 and 5 also show the central lapse function $\alpha_{\mathrm{c}}$ (dashed line; labels on the right vertical axis). In the CFC+ approach the new $h_{i j}^{\mathrm{TT}}$ terms couple directly to the metric Eq. (29) for $\alpha$, while they couple indirectly to the metric equations for $\phi$ and $\beta^{i}$ through $\alpha$ itself. The evolution of the lapse closely mirrors that of the density, decreasing while the density increases, i.e. while the star contracts, and vice versa.

For the four collapse models considered here, there is no direct visual evidence of discrepancies between the CFC and $\mathrm{CFC}+$ results. The corresponding curves for $\mathrm{CFC}$ and $\mathrm{CFC}+$ in the top panels of Figs. 4 and 5 coincide perfectly in the case of both the lapse function and the density. Therefore, as no appreciable differences are visible, we plot the relative differences $\sigma$ of these two quantities between CFC and CFC+ in the second panels (from the top) of these figures. Maximum differences of the order of $\sim 1 \%$ are found in the density evolution (solid line) for the strongly differentially rotating models A2B4G1 and A4B5G5 (see Fig. 5). In the two other models the differences are two orders of magnitude smaller. The lapse function (dashed line) shows even smaller differences between $\mathrm{CFC}$ and $\mathrm{CFC}+$, the maximum values of $\sigma$ being smaller than $0.1 \%$ even for the rapidly rotating models. This is simply due to the fact that $h_{i j}^{\mathrm{TT}}$ arises at the $2 \mathrm{PN}$ order in the 3 -metric while it appears with a pre-factor $1 / c^{6}$ in the source of $\alpha$. In fact, the relative difference roughly coincides with the 

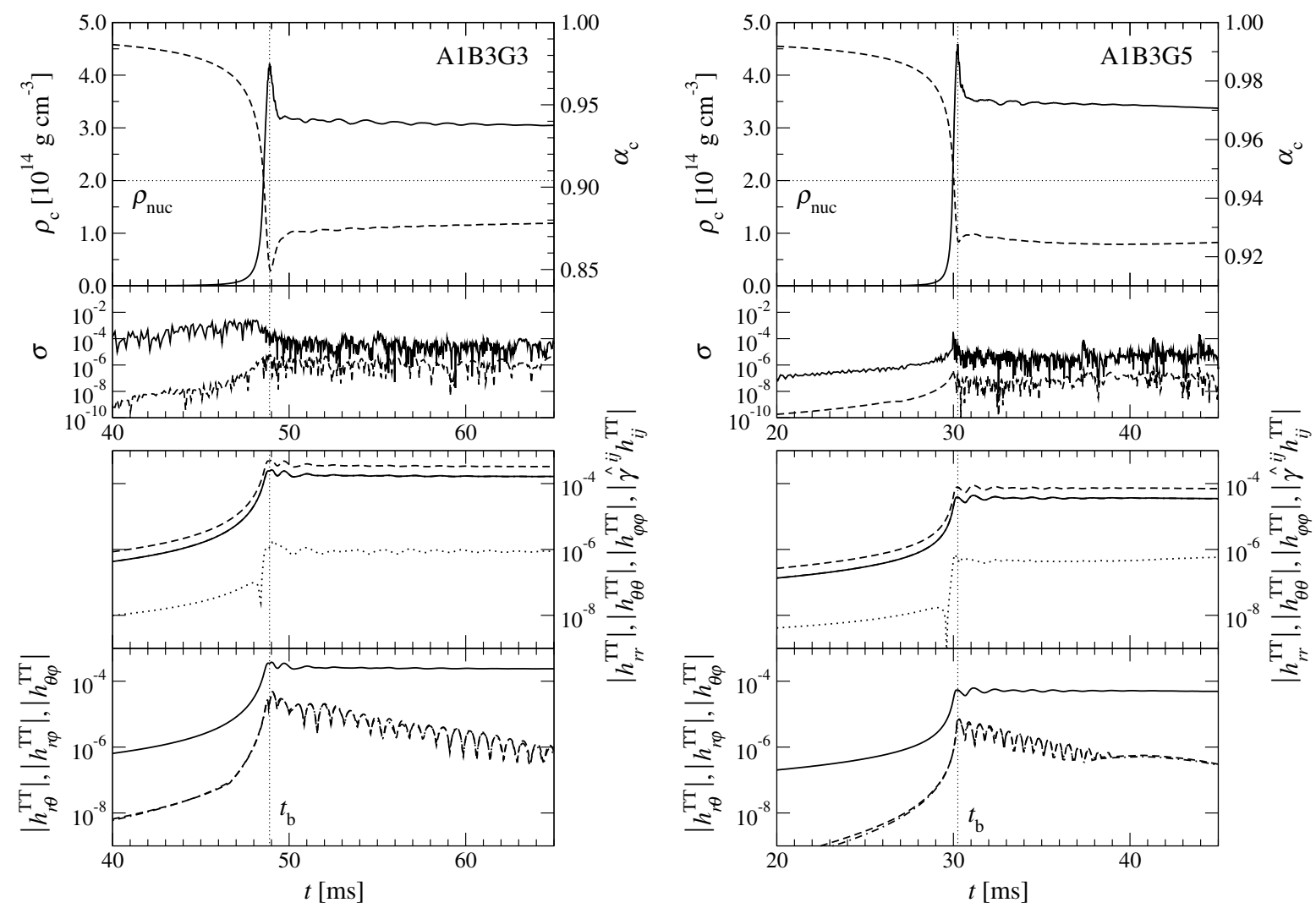

Fig. 4. Time evolution of hydrodynamic and metric quantities for the regular collapse model A1B3G3 (left) and the rapid collapse model A1B3G5 (right). The top panels show the central density $\rho_{\mathrm{c}}$ (solid line) and lapse function $\alpha_{\mathrm{c}}$ (dashed line). Both the CFC and the CFC+ results overlap. Nuclear matter density $\rho_{\text {nuc }}$ is indicated by the horizontal dotted line. The second pair of panels from the top displays the relative difference $\sigma$ of $\rho_{\mathrm{c}}$ (solid line) and $\alpha_{\mathrm{c}}$ (dashed line) between the simulation using CFC and CFC + . In the third panels the CFC+ evolution of the absolute values of $h_{r r}^{\mathrm{TT}}$ (solid line), $h_{\theta \theta}^{\mathrm{TT}}$ (dashed line), $h_{\varphi \varphi}^{\mathrm{TT}}$ (dashed-dotted line), as well as the trace of $h_{i j}^{\mathrm{TT}}$ (dotted line) are shown, all measured at the center of the star. Note that $h_{r r}^{\mathrm{TT}}$ and $h_{\varphi \varphi}^{\mathrm{TT}}$ cannot be discerned, as they practically overlap. The bottom panels depict the evolution of the maximum absolute values of $h_{r \theta}^{\mathrm{TT}}$ (solid line), $h_{r \varphi}^{\mathrm{TT}}$ (dashed line), and $h_{\theta \varphi}^{\mathrm{TT}}$ (dashed-dotted line), respectively. Again the latter two quantities closely coincide. The vertical dotted line in all panels marks the time of bounce $t_{\mathrm{b}}$.

one expected naively from the post-Newtonian approximation level, i.e., $h_{i j}^{\mathrm{TT}} \sim\left(U / c^{2}\right)$. By contrast, for slowly rotating collapses described by models A1B3G3 and A1B3G5, the nonconformally flat contribution is one order of magnitude smaller than $\left(U / c^{2}\right)$. The factor $1 / 10$ may be interpreted as coming from the proximity of spherical symmetry.

The time evolution of the diagonal components of $h_{i j}^{\mathrm{TT}}$ at the center $(r=0)$ are also plotted in Figs. 4 and 5 (third panels from the top), along with the trace of $h_{i j}^{\mathrm{TT}}$. Correspondingly, the maximum absolute values of the off-diagonal terms of $h_{i j}^{\mathrm{TT}}$ are displayed in the panels at the bottom. As expected, the various components of $h_{i j}^{\mathrm{TT}}$ appear and increase with deviations from sphericity. The profiles show that in all collapse simulations, $h_{i j}^{\mathrm{TT}}$ is quite small in comparison to the isotropic part which is of order unity. It can be seen that models with strong gravity but small asphericities (such as model A1B3G3) and models with weaker gravity but more apparent deviations from sphericity (such as models A2B4G1 or A4B5G5) all reach values for $h_{i j}^{\mathrm{TT}}$ of similar magnitude. Note that the components $h_{r \varphi}^{\mathrm{TT}}$ and $h_{\theta \varphi}^{\mathrm{TT}}$ rapidly decrease after the bounce, because a quasi-equilibrium configuration is reached in the new-born proto-neutron star. In all cases considered the trace of $h_{i j}^{\mathrm{TT}}$ is much smaller than the $h_{i j}^{\mathrm{TT}}$ components themselves, i.e. numerically $h_{i j}^{\mathrm{TT}}$ is traceless to high accuracy and also remains traceless during the entire evolution. In addition, we checked the transverse character of $h_{i j}^{\mathrm{TT}}$, i.e. $\nabla^{i} h_{i j}^{\mathrm{TT}}=0$. The latter expression is found to be compatible with zero, as the dimensionless quantity $r \nabla^{i} h_{i j}^{\mathrm{TT}}$ is much smaller than $h_{i j}^{\mathrm{TT}}$.

The radial profiles of $h_{i j}^{\mathrm{TT}}$ are very similar for all collapse models we have analyzed except for model A4B5G5 that collapses off-center with a torus-like structure. In Fig. 6 we compare this model to a model in which the maximum density is reached at the center (A1B3G3). The profiles are depicted at the instant of maximum density $\left(t_{\mathrm{b}}=48.9 \mathrm{~ms}\right.$ for model A1B3G3 and $t_{\mathrm{b}}=31.2 \mathrm{~ms}$ for model A4B5G5) and at the end of the simulation, when the system has reached equilibrium. For the spheroidal model A1B3G3, the maximum values of $h_{i j}^{\mathrm{TT}}$ are reached at the center, and the components $h_{\theta \theta}^{\mathrm{TT}}$ and $h_{\varphi \varphi}^{\mathrm{TT}}$ have local maxima inside the star. However, in the toroidal model A4B5G5 the maximum values are off-centered, while the three components exhibit their peak value inside the torus. Note that the strong deviations from sphericity in model A4B5G5 generate larger values of $h_{i j}^{\mathrm{TT}}$ as compared to model A1B3G3 at the time of bounce, but once the torus collapses to the final oblate 

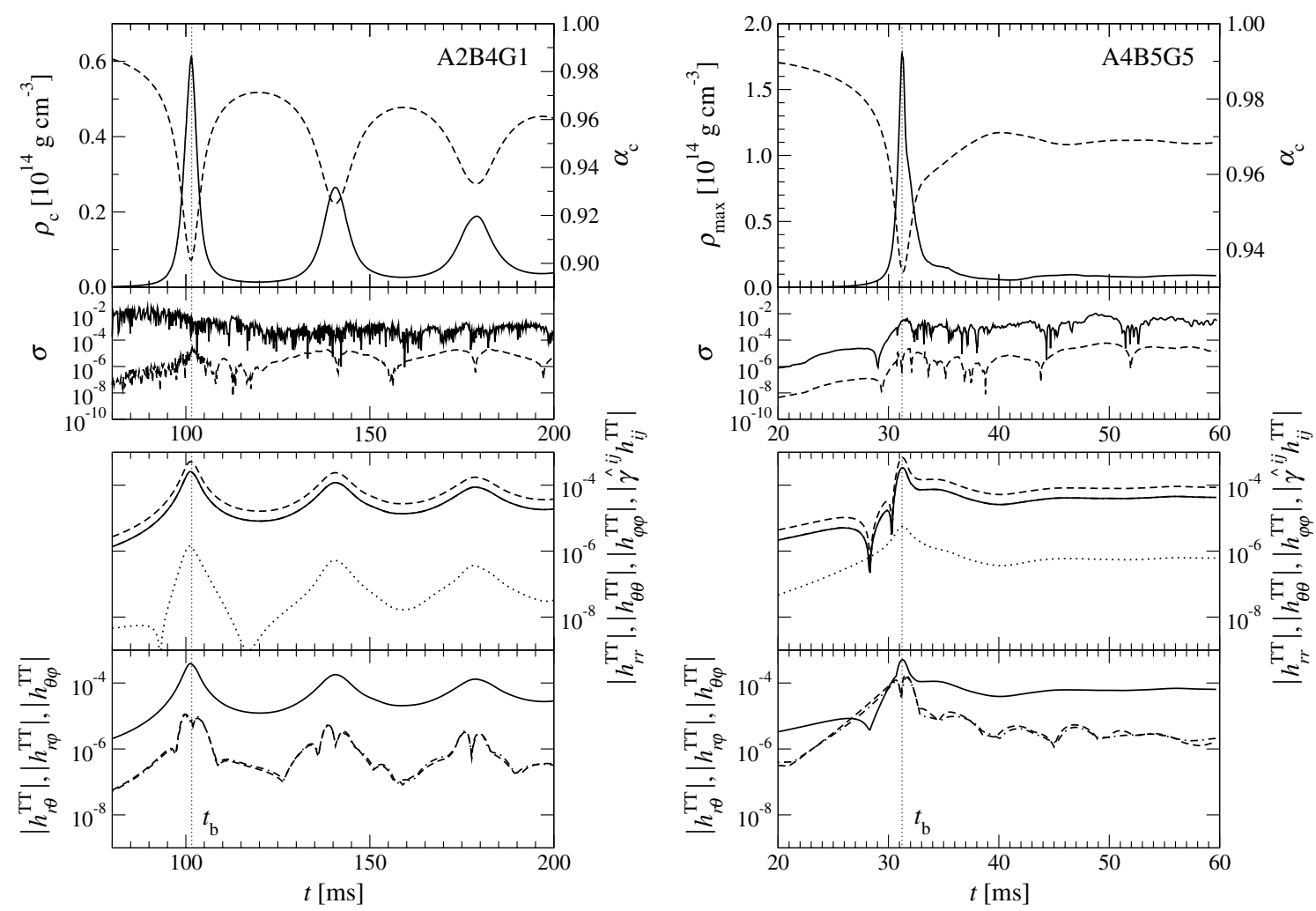

Fig. 5. Same as Fig. 4 for the multiple bounce collapse model A2B4G1 (left) and the extremely rotating collapse model A4B5G5 (right). Both models centrifugally bounce before reaching $\rho_{\text {nuc }}$. Note that for model A4B5G5 we plot the maximum density $\rho_{\max }$ instead of $\rho_{\mathrm{c}}$, as this model has a toroidal density configuration.

star, the values become smaller than for the regular collapse model.

Table 6 summarizes the results of all collapse simulations, including relevant information to calculate the size of the near zone $\lambda /(2 \pi)$ needed for the gravitational wave extraction we discuss next.

\subsection{Gravitational radiation waveforms}

Gravitational waves from the collapse simulations discussed in the preceding section were calculated for both the CFC and the $\mathrm{CFC}+$ approximation of the field equations, using the quadrupole formula. In addition, in the case of $\mathrm{CFC}+$ they were also extracted directly from $h_{i j}^{\mathrm{TT}}$ evaluated in the wave zone. The radial extension of the near zone $\lambda /(2 \pi)$ can be calculated from the approximate size of the source $r_{\mathrm{e}}$ and the typical timescale of gravitational wave emission $1 / f_{\max }$. Results for each collapse model are listed in Table 6 , which also gives the distance $r_{\mathrm{ex}}$ at which the waveforms are actually extracted from $h_{i j}^{\mathrm{TT}}$. For all models this distance is much larger than $\lambda /(2 \pi)$ (by a factor of about 50), which ensures that the wave extraction is done in the wave zone far away from the sources.

The gravitational waveforms are displayed in the top panels of Figs. 7 and 8. The waveforms extracted using the quadrupole formula (solid lines) are very similar for $\mathrm{CFC}$ and $\mathrm{CFC}+$ and would not be discernible in the figures. Thus, only the CFC+ waveforms are plotted, along with the absolute differences $\sigma_{\text {abs }}$ with respect to $\mathrm{CFC}$ (shown in the bottom panels of each figure). For all collapse models considered the differences are smaller than $0.1 \%$ of the signal maximum. This is expected from the fact that the quadrupole formula involves an integral of hydrodynamic quantities, and from the observation that the modifications in the collapse dynamics between CFC and $\mathrm{CFC}+$ are not significant, as mentioned in Sect. 6.1.

Concerning the waveforms extracted directly from $h_{i j}^{\mathrm{TT}}$ (dashed and dotted lines in Figs. 7 and 8), it can be seen that when directly using Eq. (52) to calculate the wave amplitude $A_{20}^{\mathrm{E} 2}$, the resulting signals are larger at bounce for all models. After bounce these signals show an offset for the models with stronger gravity (A1B3G3 and A1B3G5, see Fig. 7), where the waveform amplitude should actually approach zero, because the pulsations of the new-born proto-neutron star are rapidly damped and the star tends towards a state of equilibrium. If the signals are corrected by means of Eq. (54), the offset disappears and the gravitational wave amplitude agrees remarkably well with the waveforms calculated with the quadrupole amplitude. Although the extraction methods are not really independent, the agreement found between the two ways of calculating waveforms in the $\mathrm{CFC}+$ approach is a consistency check for the calculation of the $h_{i j}^{\mathrm{TT}}$, because the asymptotic behavior given by Eq. (52) can be assessed numerically this way.

The agreement holds for all cases except for model A2B4G1, where the amplitudes obtained by the quadrupole formula and from the $h_{i j}^{\mathrm{TT}}$ differ by about $50 \%$ both at the main bounce and at the subsequent bounces even after the offset 

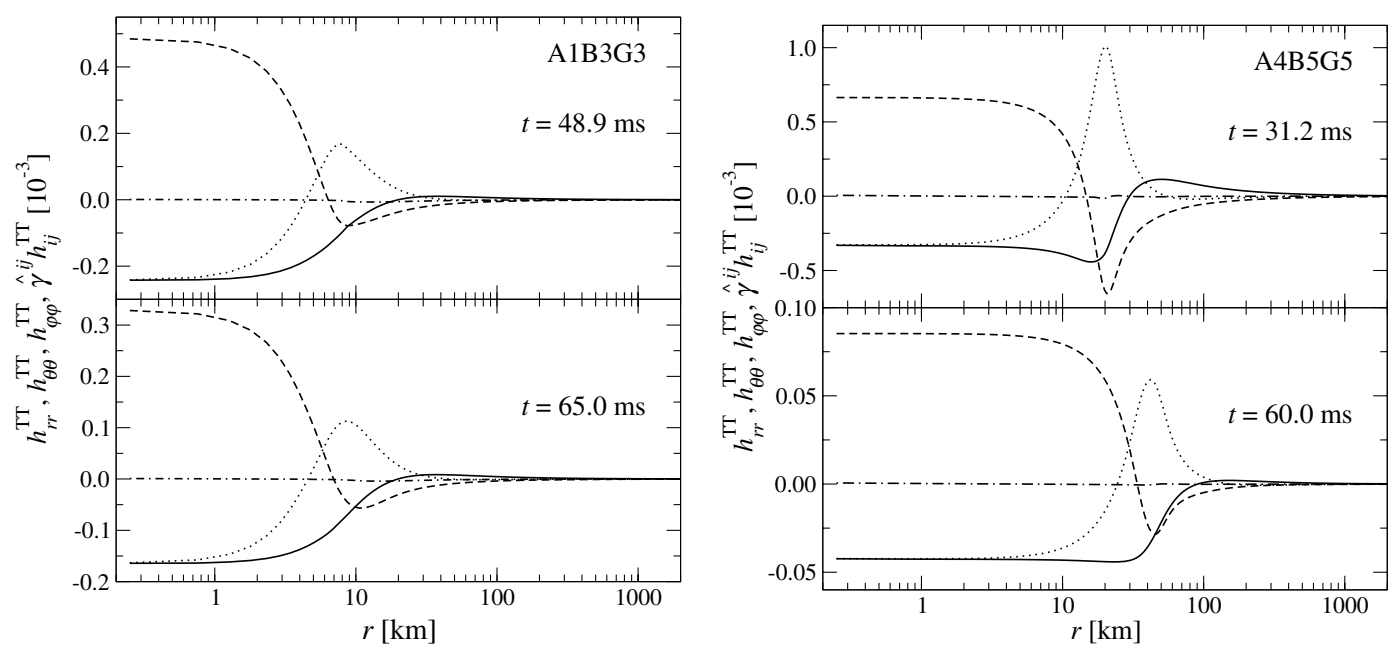

Fig. 6. Radial profiles of $h_{i j}^{\mathrm{TT}}$ at the equator for model A1B3G3 (left) and model A4B5G5 (right) at the time of maximum density (upper panels) and at the final time of the simulation (lower panels). The curves plotted correspond to $h_{r r}^{\mathrm{TT}}$ (solid line), $h_{\theta \theta}^{\mathrm{TT}}$ (dashed line), $h_{\varphi \varphi}^{\mathrm{TT}}$ (dotted line), and the trace of $h_{i j}^{\mathrm{TT}}$ (dashed-dotted line), respectively.

Table 6. Summary of various quantities that characterize the different core collapse models. The table shows the time of bounce $t_{\mathrm{b}}$, the maximum density at bounce $\rho_{\mathrm{b}}$, the maximum density reached after the bounce $\rho_{\mathrm{f}}$, the gravitational wave amplitude at bounce $\left|A_{20}^{\mathrm{E} 2}\right|_{\mathrm{b}}$ as measured using the quadrupole formula, the dominant frequency $f_{\max }$ of proto-neutron star oscillations, the radius $r_{\mathrm{ef}}$ of the star after bounce and ringdown (defined as the radial location along the equator where the density first falls below $10 \%$ of the maximum density), the size of the near zone $\lambda /(2 \pi)$, and the distance $r_{\mathrm{ex}}$ at which gravitational waves are extracted from $h_{i j}^{\mathrm{TT}}$.

\begin{tabular}{|c|c|c|c|c|c|c|c|c|}
\hline Model & $\begin{array}{c}t_{\mathrm{b}} \\
{[\mathrm{ms}]}\end{array}$ & $\begin{array}{c}\rho_{\mathrm{b}} \\
{\left[10^{14} \mathrm{~g} \mathrm{~cm}^{-3}\right]}\end{array}$ & $\begin{array}{c}\rho_{\mathrm{f}} \\
{\left[10^{14} \mathrm{~g} \mathrm{~cm}^{-3}\right]}\end{array}$ & $\begin{array}{l}\left|A_{20}^{\mathrm{E} 2}\right|_{\mathrm{b}} \\
{[\mathrm{cm}]}\end{array}$ & $\begin{array}{l}f_{\max } \\
{[\mathrm{Hz}]}\end{array}$ & $\begin{array}{c}r_{\mathrm{ef}} \\
{[\mathrm{km}]}\end{array}$ & $\begin{array}{c}\lambda /(2 \pi) \\
{[\mathrm{km}]}\end{array}$ & $\begin{array}{c}r_{\mathrm{ex}} \\
{\left[10^{3} \mathrm{~km}\right]}\end{array}$ \\
\hline A1B3G3 & 48.89 & 4.23 & 3.22 & 1223 & 674 & 13 & 71 & 2.6 \\
\hline A1B3G5 & 30.25 & 4.65 & 3.53 & 131 & 890 & 9 & 52 & 2.6 \\
\hline $\mathrm{A} 2 \mathrm{~B} 4 \mathrm{G} 1$ & 101.60 & 0.60 & 0.27 & 936 & 54 & 34 & 884 & 40.0 \\
\hline A4B5G5 & 31.23 & 1.78 & 0.096 & 3757 & 142 & 60 & 334 & 20.0 \\
\hline
\end{tabular}

correction is applied. For this particular model the size of the near zone is very large, $\lambda /(2 \pi)=884 \mathrm{~km}$; therefore, an accurate extraction of the waveforms can only be performed at a radius very far away from the star. We thus set the extraction radius $r_{\mathrm{ex}}=4 \times 10^{4} \mathrm{~km}$. As a consequence the extended grid needs to be covered with at least 600 radial zones in order to avoid too extreme logarithmic cell spacing, which would be the source of numerical inaccuracies in the fall-off behavior when solving the elliptic equations with finite difference methods.

\section{Summary and conclusions}

We have presented a new approximation for the Einstein field equations, which we call $\mathrm{CFC}+$. We tested its suitability for simulations of rotating neutron star spacetimes, both for equilibrium models and for configurations formed after gravitational core collapse. This approach is based on second order post-Newtonian corrections from conformal flatness, i.e. CFC+ represents an extension of the CFC (or Isenberg-Wilson-Mathews) approximation. The derivation of the extended system of equations has been presented in great detail, as well as the boundary conditions to apply when numerically solving them. All CFC+ field equations are elliptic, since at second post-Newtonian order the hyperbolic character of the Einstein equations disappears. This is a consequence of the fact that the time derivatives of $h_{i j}^{\mathrm{TT}}$ appear first at the 2.5 th post-Newtonian order.

We note in passing that solving elliptic equations ensures numerical stability of the solution and avoids numerical problems sometimes encountered in long-term evolutions of strongly gravitating systems when using the $3+1$ formulation of general relativity. On the other hand, the price to pay for using this approximation is that gravitational radiation reaction on the dynamics, caused by gravitational waves carrying away energy and angular momentum from the system, is absent. However, in the case of models where a comparison of our results to fully general relativistic results is possible, we checked that the absence of gravitational back-reaction does not significantly affect the results. In scenarios such as the merging of compact binaries (not investigated here), this effect would indeed be important, but only at late times. Hence, $\mathrm{CFC}+$ should also be a good approximation for modeling phenomena occurring on dynamical timescales, such as the final stages of the plunge and merger.

We compared the new CFC+ approximation with the CFC approach used by Dimmelmeier et al. $(2002 a, b)$ in two different scenarios, oscillating relativistic stars and core collapse to a neutron star. In the case of pulsating neutron stars, we find that there are no differences in the calculation of the quasi-radial 

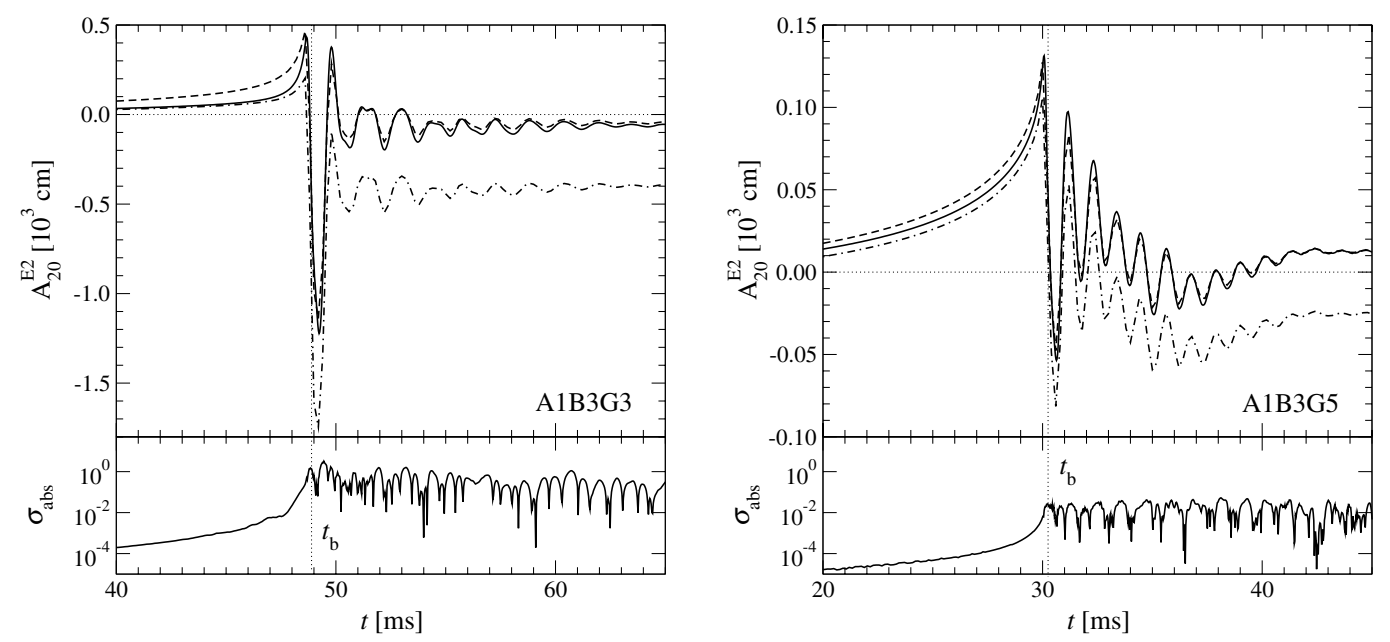

Fig. 7. Gravitational wave amplitude $A_{20}^{\mathrm{E} 2}$ computed with the $\mathrm{CFC}+$ approximation of the spacetime metric for the regular collapse model A1B3G3 (left) and the rapid collapse model A1B3G5 (right). Depicted in the top panels are the waveforms extracted using the quadrupole formula (solid line) and extracted directly from $h_{i j}^{\mathrm{TT}}$ with (dashed line) or without (dashed-dotted line) corrections for the offset after core bounce. The lower panels show the absolute difference $\sigma_{\text {abs }}$ of the quadrupole waveforms obtained using the CFC + and $\mathrm{CFC}$ approximation of the spacetime metric. The vertical dotted line marks the time of bounce $t_{\mathrm{b}}$.
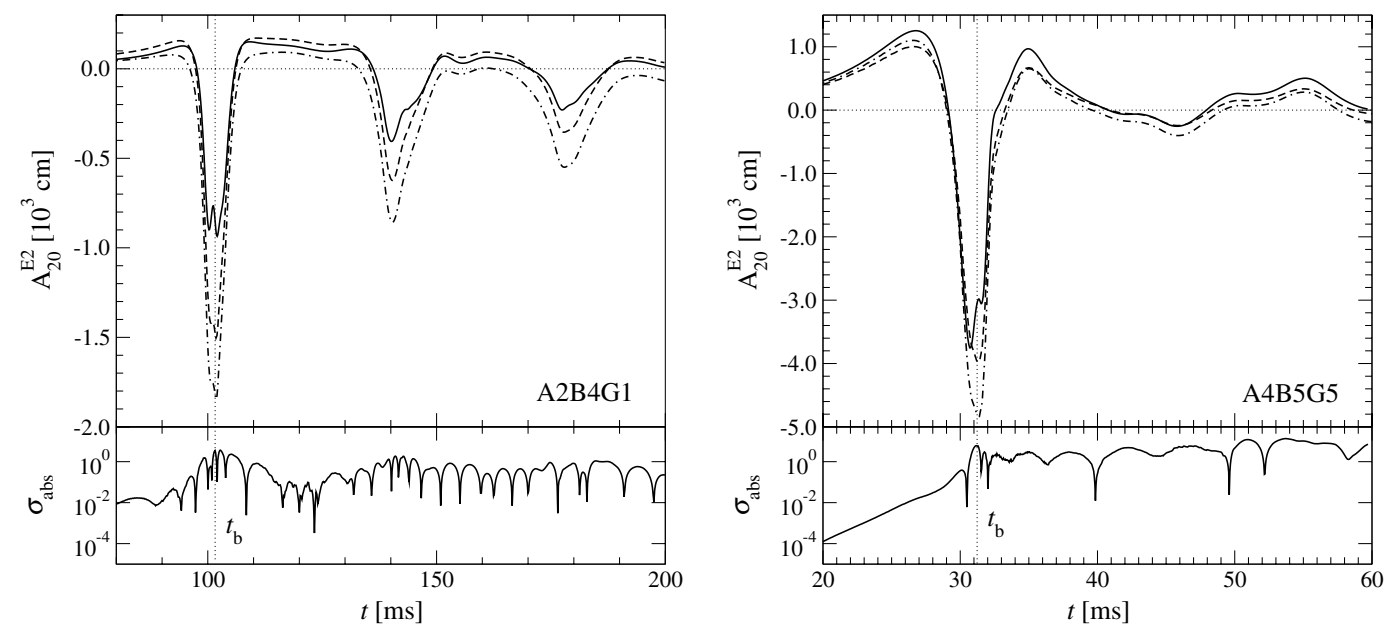

Fig. 8. Same as Fig. 7 for the multiple bounce model A2B4G1 (left) and the extremely rotating collapse model A4B5G5 (right).

normal mode frequencies of those objects, even in the most extreme situations considered when the star is rotating at the maximum allowed rate (i.e. at the mass-shedding limit). It has been possible to compare our results directly with fully general relativistic computations and, again, a very close agreement is found. Furthermore, our simulations of stellar core collapse to neutron stars covered the basic morphology and dynamics of core collapse types studied by Dimmelmeier et al. (2002b) well, including the extreme case of a core with strong differential rotation and torus-like structure. Once more, no significant differences between the two approximations are observed. Therefore, we can conclude that second post-Newtonian corrections to $\mathrm{CFC}$ do not significantly improve the results when simulating the dynamics of core collapse to a neutron star as well as when investigating the evolution of neutron stars in equilibrium.

Regarding the gravitational wave extraction we did not observe any substantial differences between CFC and CFC+ as well. The comparison has been carried out using the quadrupole formula, commonly employed in the literature to extract gravitational waveforms. In addition we also calculated the gravitational waves directly from the $h_{i j}^{\mathrm{TT}}$, which permits a straightforward use of the spacetime metric to study the gravitational wave generation mechanism from the near zone to the wave zone. Although the waveforms computed with the latter approach cannot be regarded as an independent way of calculating gravitational wave signals, it nevertheless provides a good consistency check of the CFC+ approximation that has served to validate the numerical scheme we use to calculate $h_{i j}^{\mathrm{TT}}$.

The main conclusion of this work is the assessment of the CFC approximation as a highly suitable alternative to the full Einstein equations in axisymmetric scenarios, involving rotating neutron stars in equilibrium and as end states of core collapse. These findings are supported by two facts: first, we demonstated that second post-Newtonian corrections do not play an important role in either the dynamics or the gravitational radiation waveforms of core collapse. This suggests that 
higher order post-Newtonian corrections will be completely insignificant at least on dynamic timescales. Second, the direct comparison of the CFC approach with exact fully general relativistic simulations of pulsating neutron stars yields normalmode frequencies in excellent agreement. Furthermore, comparisons of the CFC approach with fully general relativistic simulations were also reported recently by Shibata \& Sekiguchi (2004a) in the context of axisymmetric core collapse simulations. Again, the differences found in both the dynamics and the waveforms are minute, which highlights the suitability of $\mathrm{CFC}$ (and $\mathrm{CFC}+$ ) for performing accurate simulations of those scenarios without the need for solving the full system of the Einstein equations.

With this investigation we showed that a numerical code based on CFC is a very useful tool for investigating core collapse to neutron stars in general relativity. Hence, the CFC approach (as well as $\mathrm{CFC}+$ ) is suitable to form the basis of a future core collapse supernova code which can be gradually extended in various directions to incorporate additional physics such as realistic equations of state, magnetic fields, and eventually neutrino transport. In the near future we plan to further validate the $\mathrm{CFC}+$ equations in other scenarios where higher densities are present (e.g. collapse to a black hole), as well as in the fully three-dimensional case (namely to investigate the merging of neutron stars). Such scenarios are in principle beyond the range of applicability of the CFC approximation, but can possibly still be handled in a satisfactory way with the new $\mathrm{CFC}+$ approach presented in this work.

Acknowledgements. This work has been supported by the Spanish Ministerio de Ciencia y Tecnología (grant AYA 2001-3490-C0201 ), by the German Science Foundation DFG (SFB/Transregio 7 "Gravitationswellenastronomie"), and by the EU Network Programme (Research Training Network Contract HPRN-CT-2000-00137). All numerical computations were performed with the SGI Altix 3700 computer CERCA at the Departamento de Astronomía y Astrofísica of the University of Valencia.

\section{Appendix A: metric equation for the lapse function in CFC+}

The correction to the CFC metric up to second post-Newtonian order may be obtained in the Hamiltonian framework of ADM within the eponym gauge (see also Regge \& Teitelboim 1974, for an analysis in asymptotically flat spacetimes; and Holm 1985 , for a generalization to isolated perfect fluids). The original canonical variables are chosen to be the 3-metric $\gamma_{i j}$ and its conjugate momentum $c^{3} \pi^{i j} /(16 \pi G)$ with $\pi^{i j}=-\sqrt{\bar{\gamma}}\left(K^{i j}-\right.$ $K \gamma^{i j}$ ), but once the coordinate system has been fixed, four of the six remaining field degrees of freedom are eliminated by imposing the Hamiltonian constraint (14) and the three momentum constraints (15). These four field degrees of freedom correspond to the conformal factor $\phi$ and to the symmetric trace-free part $\pi_{\text {LL }}^{i j}$ of the tensor $2 \hat{\Delta}^{-1}\left(\hat{\gamma}^{i k} \hat{\nabla}_{k} \hat{\nabla}_{l} \pi^{j l}\right)-$ $\hat{\Delta}^{-2}\left(\hat{\gamma}^{i m} \hat{\gamma}^{j n} \hat{\nabla}_{k} \hat{\nabla}_{l} \hat{\nabla}_{m} \hat{\nabla}_{n} \pi^{k l}\right) / 2$, respectively.

Only two transverse trace-free (TT) field variables are left, namely $h_{i j}^{\mathrm{TT}}=\gamma_{i j}-\phi^{4} \hat{\gamma}_{i j}$ on the one hand, and $\pi_{\mathrm{TT}}^{i j}=\pi^{i j}-\pi_{\mathrm{LL}}^{i j}$ on the other. By construction, we have:

$\hat{\gamma}^{k l} \hat{\nabla}_{k} h_{i l}^{\mathrm{TT}}=0, \hat{\gamma}^{i j} h_{i j}^{\mathrm{TT}}=0$, $\hat{\nabla}_{j} \pi_{\mathrm{TT}}^{i j}=0, \hat{\gamma}_{i j} \pi_{\mathrm{TT}}^{i j}=0$.

The reduced Hamiltonian $H$ is obtained by substituting $\phi$ with $\phi\left[h_{p q}^{\mathrm{TT}}, \pi_{\mathrm{TT}}^{p q}\right]$ and $\pi^{i j}$ with $\pi^{i j}\left[h_{p q}^{\mathrm{TT}}, \pi_{\mathrm{TT}}^{p q}\right]$ in the Hamiltonian of general relativity for asymptotically flat spacetimes. The contributions of the super-Hamiltonian and super-momentum densities vanish, so that $H$ [matter variables, $\left.h_{p q}^{\mathrm{TT}}, \pi_{\mathrm{TT}}^{p q}\right]$ is given by the surface integral defining the ADM mass "on a shell",

$$
\begin{aligned}
H & =\frac{c^{4}}{16 \pi G} \int \mathrm{d}^{2} \hat{S}^{i} \sqrt{\hat{\gamma}} \gamma^{j k}\left(\hat{\nabla}_{j} \gamma_{i k}-\hat{\nabla}_{i} \gamma_{j k}\right) \\
& =-\frac{c^{4}}{2 \pi G} \int \mathrm{d}^{3} \boldsymbol{x} \sqrt{\hat{\gamma}} \hat{\Delta} \phi
\end{aligned}
$$

where the surface element $\mathrm{d} \hat{S}^{i} \sqrt{\hat{\gamma}}$ refers to the flat metric. The reduced Hamiltonian (A.3) contains the full dynamical information about the system. In particular, as shown by Regge \& Teitelboim (1974), the field evolution is governed by the equations of motion

$\partial_{t} h_{i j}^{\mathrm{TT}}=\frac{16 \pi G}{c^{3}} \hat{\gamma}_{i j}^{\mathrm{TT} k l} \frac{\delta H}{\delta \pi_{\mathrm{TT}}^{k l}}$

$\partial_{t} \pi_{\mathrm{TT}}^{i j}=-\frac{16 \pi G}{c^{3}} \hat{\gamma}_{\mathrm{TT} k l}^{i j} \frac{\delta H}{\delta h_{k l}^{\mathrm{TT}}}$,

with

$$
\begin{aligned}
\hat{\gamma}_{i j}^{\mathrm{TT} k l}= & \frac{1}{2}\left(\delta_{i}^{k}-\hat{\gamma}^{k p} \hat{\Delta}^{-1} \hat{\nabla}_{i} \hat{\nabla}_{p}\right)\left(\delta_{j}^{l}-\hat{\gamma}^{l q} \hat{\Delta}^{-1} \hat{\nabla}_{j} \hat{\nabla}_{q}\right) \\
& +\frac{1}{2}\left(\delta_{j}^{k}-\hat{\gamma}^{k p} \hat{\Delta}^{-1} \hat{\nabla}_{j} \hat{\nabla}_{p}\right)\left(\delta_{i}^{l}-\hat{\gamma}^{l q} \hat{\Delta}^{-1} \hat{\nabla}_{i} \hat{\nabla}_{q}\right) \\
& -\frac{1}{2}\left(\hat{\gamma}_{i j}-\hat{\Delta}^{-1} \hat{\nabla}_{i} \hat{\nabla}_{j}\right)\left(\hat{\gamma}^{k l}-\hat{\gamma}^{k p} \hat{\gamma}^{l q} \hat{\Delta}^{-1} \hat{\nabla}_{p} \hat{\nabla}_{q}\right),
\end{aligned}
$$

and a similar formula for $\hat{\gamma}_{\mathrm{T} k \mathrm{l}}^{i j}$. The role of these operators is to ensure the transverse trace-free projection of the Fréchet derivative $\delta H / \delta \pi_{\mathrm{TT}}^{k l}$ and $\delta H / \delta h_{k l}^{\mathrm{TT}}$, respectively. The calculation of $H$ in terms of $D^{*}, S_{i}^{*}, P$, as well as the field variables $h_{i j}^{\mathrm{TT}}$ and $\pi_{\mathrm{TT}}^{i j}$, can be done essentially by eliminating $\phi$ in Eq. (A.3) with the help of the Hamiltonian constraint (14). This is achievable in perturbative treatments such as the post-Minkowskian or the post-Newtonian ones, consisting of the formal expansion of all quantities at play in powers of the gravitational constant $G$ or of the inverse of the square of the speed of light $1 / c^{2}$.

In the course of eliminating $\phi$, we use Eq. (14) in a more explicit form. For this we first express the 3-curvature $R$ as a function of $\phi$ and $h_{i j}^{\mathrm{TT}}$. By making extensive use of the relations $\gamma_{i j} \hat{\nabla}_{l} \gamma^{j k}=-\gamma^{j k} \hat{\nabla}_{l} \gamma_{i j}$ and $\hat{\nabla}_{k} \bar{\gamma}=\left(\bar{\gamma} \gamma^{i j}\right) \hat{\nabla}_{k} \gamma_{i j}$, the combination $\bar{\gamma}^{3} R$ can be written as (cf. Schwinger 1963)

$$
\begin{aligned}
\bar{\gamma}^{3} R= & -\bar{\gamma}^{2} \hat{\nabla}_{i} \hat{\nabla}_{j}\left(\bar{\gamma} \gamma^{i j}\right) \\
& +\frac{1}{2} \hat{\nabla}_{i} \bar{\gamma}^{2} \hat{\nabla}_{j}\left(\bar{\gamma} \gamma^{i j}\right)-\frac{1}{4}\left(\bar{\gamma} \gamma^{i j}\right) \hat{\nabla}_{i} \bar{\gamma} \hat{\nabla}_{j} \bar{\gamma} \\
& -\frac{1}{2} \bar{\gamma}\left(\bar{\gamma} \gamma^{j l}\right) \hat{\nabla}_{j}\left(\bar{\gamma} \gamma^{i k}\right) \hat{\nabla}_{i} \gamma_{k l} \\
& +\frac{1}{2}\left(\bar{\gamma} \gamma^{i k}\right)\left(\bar{\gamma} \gamma^{j l}\right) \hat{\nabla}_{j} \bar{\gamma} \hat{\nabla}_{i} \gamma_{k l} \\
& +\frac{1}{4} \bar{\gamma}\left(\bar{\gamma} \gamma^{i k}\right) \hat{\nabla}_{k}\left(\bar{\gamma} \gamma^{j l}\right) \hat{\nabla}_{i} \gamma_{j l} \\
& -\frac{1}{4}\left(\bar{\gamma} \gamma^{j l}\right)\left(\bar{\gamma} \gamma^{i k}\right) \hat{\nabla}_{k} \bar{\gamma} \hat{\nabla}_{i} \gamma_{j l} .
\end{aligned}
$$


By definition, the determinant $\bar{\gamma}$ is equal to the antisymmetric sum of products $3 ! \hat{\gamma}^{p[1} \hat{\gamma}^{2 q} \hat{\gamma}^{3] r} \gamma_{p 1} \gamma_{q 2} \gamma_{r 3}=\hat{\gamma}^{p[i} \hat{\gamma}^{j q} \hat{\gamma}^{k] r} \gamma_{p i} \gamma_{q j} \gamma_{r k}$ on a Cartesian grid, the square brackets denoting antisymmetrization of non-underlined indices. Its explicit expression is given, e.g., by Schäfer (1985). Similarly, the $i j$-component of the comatrix $\bar{\gamma} \gamma^{i j}$ is known to be $\varepsilon^{i k l}\left(\varepsilon_{r m n} \hat{\gamma}^{j r} \hat{\gamma}^{m p} \hat{\gamma}^{n q} \gamma_{p k} \gamma_{q l}\right) / 2$, where $\varepsilon^{i k l}$ is the permutation operator. From the identity $\varepsilon^{r k l} \varepsilon_{j m n}=3 ! \delta_{j}^{[r} \delta_{m}^{k} \delta_{n}^{l]}$ it is straightforward to obtain $\hat{\gamma}_{i k} \hat{\gamma}_{j l} \bar{\gamma} \hat{\gamma}^{k l}$ as a function of $\phi$ and $h_{i j}^{\mathrm{TT}}$. After inserting the relations for $\bar{\gamma}$ and $\bar{\gamma} \gamma^{i j}$ in the right-hand side of Eq. (A.7), it is expanded in powers of $h_{i j}^{\mathrm{TT}}$ and truncated consistently at the post-Newtonian level of $h_{i j}^{\mathrm{TT}} h_{k l}^{\mathrm{TT}}$, denoted as $O\left(h^{2}\right)$. At this point we have an expression for $\bar{\gamma} R$ as a function of $\hat{\Delta} \phi$.

In addition to $R$, there is a second contribution to the lefthand side of the Hamiltonian constraint surviving in the absence of matter, namely $K_{i j} K^{i j}-K^{2}=\left[\pi_{j}^{i} \pi_{i}^{j}-\frac{1}{2}\left(\pi_{i}^{i}\right)^{2}\right] / \bar{\gamma}$. In the ADM formulation, the momentum $\pi^{i j}$ decomposes into $\pi_{\mathrm{LL}}^{i j}+\pi_{\mathrm{TT}}^{i j}$. The first term $\pi_{\mathrm{LL}}^{i j}$ is of order $1 / c^{3}$, being a sum of derivatives of Poisson inverse operators acting on $\hat{\nabla}_{j} \pi^{i j}=$ $O\left(\nabla_{j} K^{i j}\right)$, which is itself $O\left(1 / c^{3}\right)$ according to the momentum constraint equation. The second term, linear in $h_{i j}^{\mathrm{TT}}$, is transverse and trace-free, hence $\hat{\gamma}_{i j} \pi_{\mathrm{TT}}^{i j}=0$. Moreover, the ADM gauge condition implies $\hat{\gamma}_{i j} \pi^{i j}=\hat{\gamma}_{i j} \pi_{\mathrm{LL}}^{i j}=0$.

Finally, we consider the matter source term $E$ in the Hamiltonian constraint. The corresponding density $E^{*}=\sqrt{\bar{\gamma}} E$ may be written as

$$
\begin{aligned}
\frac{16 \pi G}{c^{4}} E^{*}= & \frac{16 \pi G}{c^{2}}\left\{\left[\left(D^{*} h\right)^{2}+\phi^{-4} \hat{\gamma}^{m n} \frac{S_{m}^{*} S_{n}^{*}}{c^{2}}\right]^{1 / 2}\right. \\
& \left.\times\left(1-\frac{1}{2} \hat{\gamma}^{i k} \hat{\gamma}^{j l} h_{k l}^{\mathrm{TT}} \frac{S_{i}^{*} S_{j}^{*}}{c^{2} D^{* 2}}\right)-\frac{\phi^{6} P}{c^{2}}\right\} \\
& +O\left(\frac{h}{c^{6}}\right)+O\left(\frac{h^{2}}{c^{2}}\right) .
\end{aligned}
$$

This yields an elliptic equation for $V=2(\phi-1)=O\left(1 / c^{2}\right)$ up to $O\left(h / c^{6}\right)$ and $O\left(h^{2} / c^{2}\right)$ corrections:

$$
\begin{aligned}
-4 \hat{\Delta} V= & -4 \hat{\nabla}_{i} \phi \hat{\nabla}_{j} \phi \hat{\gamma}^{i k} \hat{\gamma}^{j l} h_{k l}^{\mathrm{TT}} \\
& +\frac{1}{4} \hat{\gamma}^{k l} \hat{\gamma}^{i m} \hat{\gamma}^{j n} \hat{\nabla}_{k} h_{i j}^{\mathrm{TT}} \hat{\nabla}_{l} h_{m n}^{\mathrm{TT}} \\
& -4 \hat{\nabla}_{i}\left(\phi^{-4} \hat{\nabla}_{j} \phi \hat{\gamma}^{i k} \hat{\gamma}^{j l} h_{k l}^{\mathrm{TT}}\right)-\frac{1}{2} \hat{\Delta}\left(\hat{\gamma}^{i j} \hat{\gamma}^{k l} h_{i k}^{\mathrm{TT}} h_{j l}^{\mathrm{TT}}\right) \\
& +\frac{1}{2} \hat{\nabla}_{i} \hat{\nabla}_{j}\left(\hat{\gamma}^{k l} \hat{\gamma}^{m i} \hat{\gamma}^{n j} h_{k m}^{\mathrm{TT}} h_{l n}^{\mathrm{TT}}\right) \\
& +\hat{\gamma}_{i k} \hat{\gamma}_{j l}\left(\pi_{\mathrm{LL}}^{i j} \pi_{\mathrm{LL}}^{k l}+2 \pi_{\mathrm{LL}}^{i j} \pi_{\mathrm{TT}}^{k l}+\pi_{\mathrm{TT}}^{i j} \pi_{\mathrm{TT}}^{k l}\right) \\
& +\frac{16 \pi G}{c^{2}} \phi^{-1}\left\{\left(D^{*} h\right)^{2}+\phi^{-4} \hat{\gamma}^{m n} \frac{S_{m}^{*} S_{n}^{*}}{c^{2}}\right]^{1 / 2} \\
& \left.\times\left(1-\frac{1}{2} \hat{\gamma}^{i k} \hat{\gamma}^{j l} h_{k l}^{\mathrm{TT}} \frac{S_{i}^{*} S_{j}^{*}}{c^{2} D^{* 2}}\right)-\frac{\phi^{6} P}{c^{2}}\right\} \\
& +O\left(\frac{h}{c^{6}}\right)+O\left(\frac{h^{2}}{c^{2}}\right) .
\end{aligned}
$$

As the terms containing a factor $h_{i j}^{\mathrm{TT}}$ or $\pi_{\mathrm{TT}}^{i j}$ are proportional to the coupling constant $G / c^{2}$ of general relativity, Eq. (A.9) reduces to $\hat{\Delta} V=-4 \pi G D^{*} / c^{2}+O\left(1 / c^{4}\right)$ at the lowest post-Newtonian approximation. Thus, if we introduce the Newtonian potential $U$ defined as the smooth solution of the Poisson Eq. (28) vanishing at spatial infinity, we have $V=U / c^{2}+O\left(1 / c^{4}\right)$, plus a possible harmonic function. Assuming an asymptotically flat space-time, this function must tend asymptotically towards zero while being regular, and so it has to be identically zero. Another important piece of information provided by Eq. (A.9) is the value of the lowest order contribution to the potential $V$ that depends on the field variables. It is given by the equation

$$
\begin{aligned}
-4 V= & \hat{\Delta}^{-1}\left(-4 \hat{\nabla}_{i} \hat{\nabla}_{j} \phi \hat{\gamma}^{i k} \hat{\gamma}^{j l} h_{k l}^{\mathrm{TT}}\right)+O\left(\frac{h}{c^{4}}\right) \\
& + \text { pure matter part, }
\end{aligned}
$$

which shows incidentally that $\phi=1+V / 2$ is not affected by a non-zero $h_{i j}^{\mathrm{TT}}$ at the leading post-Newtonian approximation in the field. Inserting the resulting expression for the conformal factor into the Hamiltonian constraint (A.9), we arrive at

$$
\begin{aligned}
-4 \hat{\Delta} V= & -\frac{1}{c^{4}}\left(2 \hat{\nabla}_{i} U \hat{\nabla}_{j} U+8 \pi G \frac{S_{i}^{*} S_{j}^{*}}{D^{*}}\right) \hat{\gamma}^{i k} \hat{\gamma}^{j l} h_{k l}^{\mathrm{TT}} \\
& +\frac{1}{4} \hat{\gamma}^{k l} \hat{\gamma}^{i m} \hat{\gamma}^{j n} \hat{\nabla}_{k} h_{i j}^{\mathrm{TT}} \hat{\nabla}_{l} h_{m n}^{\mathrm{TT}} \\
& +\hat{\gamma}_{i k} \hat{\gamma}_{j l}\left(2 \pi_{\mathrm{LL}}^{i j} \pi_{\mathrm{TT}}^{k l}+\pi_{\mathrm{TT}}^{i j} \pi_{\mathrm{TT}}^{k l}\right) \\
& +O\left(\frac{h}{c^{6}}\right)+O\left(\frac{h^{2}}{c^{2}}\right) \\
& + \text { total derivative }+ \text { pure matter part. }
\end{aligned}
$$

The pure matter part has been kept aside because it does not enter the computation of $h_{i j}^{\mathrm{TT}}$. The total derivative terms are irrelevant for the investigation of the field evolution. Indeed, by virtue of relation (A.3), the Hamiltonian is given by the spatial integral of $-c^{4} \hat{\Delta} V /(4 \pi G)$. Thus the dynamics of the gravitational interaction is described by

$$
\begin{aligned}
H_{\substack{\text { field } \\
\text { +int. }}}= & \frac{c^{4}}{16 \pi G} \int \mathrm{d}^{3} \boldsymbol{x} \sqrt{\hat{\gamma}} \\
& \times\left[-\frac{1}{c^{4}}\left(2 \hat{\nabla}_{i} U \hat{\nabla}_{j} U+8 \pi G \frac{S_{i}^{*} S_{j}^{*}}{D^{*}}\right) \hat{\gamma}^{i k} \hat{\gamma}^{j l} h_{k l}^{\mathrm{TT}}\right. \\
& -\frac{1}{4} \hat{\Delta}\left(\hat{\gamma}^{i m} \hat{\gamma}^{j n} h_{m n}^{\mathrm{TT}}\right) h_{i j}^{\mathrm{TT}} \\
& \left.+\hat{\gamma}_{i k} \hat{\gamma}_{j l}\left(2 \pi_{\mathrm{LL}}^{i j} \pi_{\mathrm{TT}}^{k l}+\pi_{\mathrm{TT}}^{i j} \pi_{\mathrm{TT}}^{k l}\right)\right] \\
& +O\left(\frac{h}{c^{6}}\right)+O\left(\frac{h^{2}}{c^{2}}\right)
\end{aligned}
$$


in agreement with Schäfer (1990). The Hamilton equations provide the evolution of the field. They take the explicit form

$$
\begin{aligned}
\partial_{t} h_{i j}^{\mathrm{TT}}= & 2 c \hat{\gamma}_{i j}^{\mathrm{TT} k l}\left[\hat{\gamma}_{k m} \hat{\gamma}_{l n}\left(\pi_{\mathrm{LL}}^{m n}+\pi_{\mathrm{TT}}^{m n}\right)\right] \\
& +O\left(\frac{1}{c^{5}}\right)+O\left(\frac{h}{c}\right) \\
= & 2 c \hat{\gamma}_{i k} \hat{\gamma}_{j l} \pi_{\mathrm{TT}}^{k l}+O\left(\frac{1}{c^{5}}\right)+O\left(\frac{h}{c}\right), \\
\partial_{t} \pi_{\mathrm{TT}}^{i j}= & -c \hat{\gamma}_{\mathrm{TT} k l}^{i j}\left[-\frac{2}{c^{4}}\left(\hat{\nabla}_{m} U \hat{\nabla}_{n} U+4 \pi G \frac{S_{m}^{*} S_{n}^{*}}{D^{*}}\right) \hat{\gamma}^{k m} \hat{\gamma}^{l n}\right. \\
& \left.-\frac{1}{2} \hat{\Delta}\left(\hat{\gamma}^{k m} \hat{\gamma}^{l n} h_{m n}^{\mathrm{TT}}\right)\right] \\
& +O\left(\frac{1}{c^{5}}\right)+O\left(\frac{h}{c}\right) .
\end{aligned}
$$

The non-conformally flat part of the 3-metric appears first at the second post-Newtonian approximation. Its leading order is obtained by inserting the above expression for $\partial_{t} \pi_{\mathrm{TT}}^{i j}$ into the time derivative of Eq. (A.13). The resulting equation is of wave type. In the near zone, all terms of order $1 / c^{6}$ may be neglected, in particular the time derivative contribution to the d'Alembertian operator. Hence the non-conformally flat part of the 3-metric satisfies

$\hat{\Delta} h_{i j}^{\mathrm{TT}}=-\frac{\hat{\gamma}_{i j}^{\mathrm{TT} k l}}{c^{4}}\left(4 \hat{\nabla}_{k} U \hat{\nabla}_{l} U+16 \pi G \frac{S_{k}^{*} S_{l}^{*}}{D^{*}}\right)+O\left(\frac{1}{c^{6}}\right)$,

which is identical up to second post-Newtonian order to Eq. (26).

The equation for the lapse function $\alpha$ is derived from the gauge conditions (17), (18) combined with the evolution Eq. (13) for the extrinsic curvature $K_{i j}$. From the identity $\pi^{i j} \hat{\gamma}_{i j}=0$, we deduce that the trace is negligible at this level of approximation:

$$
\begin{aligned}
\hat{\gamma}^{i j} K_{i j} & =\frac{1}{2 \sqrt{\bar{\gamma}}}\left(2 \sqrt{\bar{\gamma}} \hat{\gamma}_{i j} K^{i j}\right)+O\left(\frac{h}{c^{3}}\right) \\
& =\frac{1}{2 \sqrt{\bar{\gamma}}} \pi^{i j} \hat{\gamma}_{i j}+O\left(\frac{h}{c^{3}}\right) \\
& =O\left(\frac{1}{c^{7}}\right) .
\end{aligned}
$$

By contracting Eq. (13) with the inverse 3-metric $\gamma^{i j}$, we arrive at

$$
\frac{\gamma^{i j}}{c} \partial_{t} K_{i j}=\frac{\phi^{-4}}{c} \partial_{t}\left(\hat{\gamma}^{i j} K_{i j}\right)+O\left(\frac{1}{c^{8}}\right)=O\left(\frac{1}{c^{8}}\right),
$$

from which follows that

$$
\begin{array}{r}
-\nabla_{i} \nabla^{i} \alpha+\alpha R+K^{i k}\left(-2 \alpha K_{i k}+2 \nabla_{i} \beta_{k}\right) \\
-\frac{4 \pi G}{c^{4}}(-S+3 E)=O\left(\frac{1}{c^{8}}\right) .
\end{array}
$$

Due to the fact that $K^{i k}$ is symmetric and trace-free, neglecting corrections of order $O\left(h / c^{3}\right)$, the product $K^{i k}\left(-2 \alpha K_{i k}+2 \nabla_{i} \beta_{k}\right)$ can be written as $K^{i k}\left(-2 \alpha K_{\langle i k\rangle}+2 \nabla_{\langle i} \beta_{k\rangle}\right)+O\left(h / c^{6}\right)$. The terms inside the parentheses vanish according to the symmetric tracefree version of Eq. (12),

$-2 \alpha K_{\langle i j\rangle}+2 \nabla_{\langle i} \beta_{j\rangle}=\frac{1}{c} \partial_{t} \gamma_{\langle i j\rangle}=O\left(\frac{1}{c^{5}}\right)$,

so that $K^{i k}\left(-2 \alpha K_{i k}+2 \nabla_{i} \beta_{k}\right)=O\left(1 / c^{8}\right)$. Next, we see from the Hamiltonian constraint equation that the interaction and field parts of the scalar curvature $R$ appearing in Eq. (A.18) are actually of order $O\left(h / c^{4}\right)=O\left(1 / c^{8}\right)$. On the other hand, we know that $E^{*}=O\left(h / c^{4}\right)+$ pure matter terms. We have similar equalities for $E$ and $S=S_{i}^{i}$. Therefore, we find

$$
\begin{aligned}
\frac{1}{\sqrt{\gamma}} \hat{\nabla}_{i}\left(\sqrt{\bar{\gamma}} \gamma^{i j} \hat{\nabla}_{j} \alpha\right) & =O\left(\frac{1}{c^{8}}\right)+\text { pure matter part } \\
& =\hat{\Delta} \alpha-\hat{\nabla}_{i} \hat{\nabla}_{j} \alpha \hat{\gamma}^{i k} \hat{\gamma}^{j l} h_{k l}^{\mathrm{TT}} .
\end{aligned}
$$

At the lowest approximation, we may replace $\alpha$ by $\left(-g_{00}+\right.$ $\left.\beta_{i} \beta^{i}\right)^{1 / 2}=1-U / c^{2}+O\left(1 / c^{4}\right)$. In the end, the elliptic equation for the lapse in the presence of $h_{i j}^{\mathrm{TT}}$ is modified to

$\hat{\Delta} \alpha=(\hat{\Delta} \alpha)_{h_{i j}^{\mathrm{TT}}=0}-\frac{1}{c^{2}} \hat{\gamma}^{i k} \hat{\gamma}^{j l} h_{i j}^{\mathrm{TT}} \hat{\nabla}_{k} \hat{\nabla}_{l} U+O\left(\frac{1}{c^{8}}\right)$.

This is the desired CFC+ metric equation for the lapse function $\alpha$. It can easily be transformed into Eq. (29) in Sect. 2.4, remembering that $\phi$ coincides with the CFC conformal factor at this level. Since $U / c^{2}$ is Newtonian, $\hat{\gamma}^{i k} \hat{\gamma}^{j l} h_{i j}^{\mathrm{TT}} \hat{\nabla}_{k} \hat{\nabla}_{l} U / c^{2}$ corresponds to the second post-Newtonian order for the dynamics.

The equation for the shift can be obtained in principle by contracting the 3-metric evolution with the help of the Euclidean metric $\hat{\gamma}^{i j}$. However, with respect to the conformally flat case, the new terms are proportional to a product of the type $K\left(\right.$ or $\beta$ ) times $h_{i j}^{\mathrm{TT}}$. They are therefore negligible at the second post-Newtonian level and will not be computed here. As already pointed out, the equation for the conformal factor remains unaffected at that level as well. Thus in general, all $\mathrm{CFC}$ equations, except the one for the lapse function, remain unaltered.

\section{Appendix B: inversion of the equation for the 3-metric correction in CFC+}

To express the second post-Newtonian correction $h_{i j}^{\mathrm{TT}}$ in CFC+ with the help of intermediate potentials and thus to invert Eqs. (26) into (35) in Sect. 2.4, we proceed in three stages: (i) We make the action of $\hat{\gamma}_{i j}^{\mathrm{TT} k l}$ explicit in Eq. (26). The result is integrated formally by means of the Poisson integral operator $\hat{\Delta}^{-1}$. By virtue of its linearity property, we obtain a weighted sum of Poisson potentials of generic type $\hat{\Delta}^{-1} F_{m n}$ (up to possible index contractions) or super-potentials of the form $\hat{\Delta}^{-2} \hat{\nabla}_{i} \hat{\nabla}_{j} F_{m n}=\hat{\Delta}^{-1}\left(\hat{\Delta}^{-1} \hat{\nabla}_{i} \hat{\nabla}_{j} F_{m n}\right)$ and $\hat{\Delta}^{-3} \hat{\nabla}_{i} \hat{\nabla}_{j} \hat{\nabla}_{k} F_{m n}$. (ii) We transform the super-potentials into simple Poisson potentials in order to get rid of all derivatives acting directly on the sources. (iii) We insert the resulting quantities into the transverse traceless tensor $\hat{\Delta}^{-1} \hat{\gamma}_{i j}^{\mathrm{TT} k l} F_{k l}$ and perform some additional manipulations that lead to the final expression. These steps are performed in detail in the following.

It is straightforward to expand the operator $\hat{\gamma}_{i j}^{\mathrm{TT} k l}$ defined in Eq. (A.6) and to apply it to the source $F_{k l}$ given in Eq. (27). 
Taking the symmetry in the two indices $k$ and $l$ into account, inverting the tensor Poisson Eq. (26) then yields

$$
\begin{aligned}
h_{i j}^{\mathrm{TT}}= & \hat{\Delta}^{-1}\left(\hat{\gamma}_{i j}^{\mathrm{TTkl}} F_{k l}\right) \\
= & \hat{\Delta}^{-1} F_{i j}-\frac{1}{2} \hat{\gamma}_{i j} \hat{\Delta}^{-1}\left(\hat{\gamma}^{k l} F_{k l}\right) \\
& -2 \hat{\Delta}^{-2}\left(\hat{\gamma}^{k l} \hat{\nabla}_{k} \hat{\nabla}_{i} F_{j) l}\right)+\frac{1}{2} \hat{\Delta}^{-2} \hat{\nabla}_{i} \hat{\nabla}_{j}\left(\hat{\gamma}^{k l} F_{k l}\right) \\
& +\frac{1}{2} \hat{\gamma}_{i j} \hat{\Delta}^{-2} \hat{\nabla}_{k} \hat{\nabla}_{l}\left(\hat{\gamma}^{m k} \hat{\gamma}^{n l} F_{m n}\right) \\
& +\frac{1}{2} \hat{\Delta}^{-3} \hat{\nabla}_{i} \hat{\nabla}_{j} \hat{\nabla}_{k} \hat{\nabla}_{l}\left(\hat{\gamma}^{m k} \hat{\gamma}^{n l} F_{m n}\right) .
\end{aligned}
$$

As the Poisson integral $\hat{\Delta}^{-1} F_{k l}$ converges, all other (super-)potentials entering Eq. (B.1) are also well defined. However, they cannot be handled easily. For instance, quantities such as $\hat{\Delta}^{-2} F_{m n}$ or $\hat{\Delta}^{-3} F_{m n}$ are a priori meaningless, which shows that the derivatives cannot commute with the integrals. In order to operate on the sources without meeting any serious restrictions, it is convenient to resort to the tool of Hadamard finite part regularization.

It is beyond the scope of this paper to review all properties of the Hadamard finite part. However, as it will be used intensively in the following, we recall its definition for completeness, as well as those features needed in our derivation. When a function $f(x), x \in \mathbb{R}^{3}$, is smooth outside a finite number of singularities, locally integrable, but not integrable on $\mathbb{R}^{3}$, we can instead consider the new integrand $\left(\left|\boldsymbol{x}-\boldsymbol{x}_{0}\right| / r_{0}\right)^{B} f(\boldsymbol{x})$, where $B$ is a complex number, $r_{0}$ a positive number, and where $\left|\boldsymbol{x}-\boldsymbol{x}_{0}\right|$ denotes the Euclidean norm of $\boldsymbol{x}-\boldsymbol{x}_{0}$, with $\boldsymbol{x}_{0}$ being an arbitrary vector of $\mathbb{R}^{3}$. The integral $\int_{\left|x-x_{0}\right|>r_{0}} \mathrm{~d}^{3} \boldsymbol{x}\left(\left|\boldsymbol{x}-\boldsymbol{x}_{0}\right| / r_{0}\right)^{B} f(\boldsymbol{x})$, defined by means of the natural measure $\mathrm{d}^{3} \boldsymbol{x}=\mathrm{d} x^{1} \mathrm{~d} x^{2} \mathrm{~d} x^{3}$ in Cartesian coordinates, converges for $B$ belonging to an appropriate domain $D$ of the complex plane. It can be regarded as a holomorphic function on $D$. It is then possible to extend $I_{f}(B)=\int \mathrm{d}^{3} \boldsymbol{x}\left(\left|\boldsymbol{x}-\boldsymbol{x}_{0}\right| / r_{0}\right)^{B} f(\boldsymbol{x})$ by analytic continuation as close to the point $B=0$ as desired, and to obtain its Laurent expansion $\sum_{k \in \mathbb{Z}}\left(I_{f}\right)_{k} B^{k}$ there, as explained by Blanchet \& Damour (1986). The zeroth order coefficient $\left(I_{f}\right)_{0}$ is often referred to as the finite part integral of $f(\boldsymbol{x})$, which in our notation reads

$\mathrm{FP}_{B=0} \int \mathrm{d}^{3} \boldsymbol{x} \sqrt{\hat{\gamma}}\left(\frac{\left|\boldsymbol{x}-\boldsymbol{x}_{0}\right|}{r_{0}}\right)^{B} f(\boldsymbol{x})=\left(I_{f}\right)_{0}$.

The finite part integral of $f$ may depend on the arbitrary radius $r_{0}$; in fact, this will typically happen when the result contains logarithms. Nonetheless, as long as $f$ is integrable, its finite part integral coincides with $\int \mathrm{d}^{3} \boldsymbol{x} f$ and does not show any dependence on $r_{0}$.

An important property of the Hadamard regularization is that the finite part Poisson integral of a smooth function always exists, whereas the simple Poisson integral may not. The covariant expression of this finite part reads

$\mathrm{FP} \hat{\Delta}_{\boldsymbol{x}_{0}}^{-1} f=\mathrm{FP}_{B=0} \int \frac{\mathrm{d}^{3} \boldsymbol{x}^{\prime} \sqrt{\hat{\gamma}}}{-4 \pi}\left(\frac{\left|\boldsymbol{x}^{\prime}-\boldsymbol{x}_{0}\right|}{r_{0}}\right)^{B} \frac{f\left(\boldsymbol{x}^{\prime}\right)}{\left|\boldsymbol{x}-\boldsymbol{x}^{\prime}\right|}$,

where the Euclidean volume element has been written as $\mathrm{d}^{3} \boldsymbol{x} \sqrt{\hat{\gamma}}$ in an arbitrary coordinate system. When $\hat{\Delta}^{-1} f$ exists, it satisfies FP $\hat{\Delta}_{\boldsymbol{x}_{0}}^{-1} f=\hat{\Delta}^{-1} f$. In any case, the regularized Poisson integral is the particular solution of a Poisson equation of the type $\hat{\Delta} g=f$. Thus, the operator $\operatorname{FP} \hat{\Delta}^{-1}$ constitutes a genuine generalization of the ordinary Poisson operator $\hat{\Delta}^{-1}$ and will be denoted as $\hat{\Delta}_{x_{0}}^{-1}$ henceforth. It has the important property that it commutes with the spatial derivatives $\hat{\nabla}_{i}$, which allows us to work on the form of the elementary (super-)potentials by applying simple and systematic rules.

An important formula related to the Hadamard regularization is the one giving the generalized Poisson integral of the distance to the field point $\boldsymbol{x}$,

$\hat{\Delta}_{x}^{-1}\left|x-x^{\prime}\right|^{A}=\frac{\left|x-x^{\prime}\right|^{A+2}}{(A+2)(A+3)}$

for an arbitrary complex exponent $A \neq-2,-3$. Notably, it can be used to evaluate the action of the operator $\hat{\Delta}_{x}^{-1}=\mathrm{FP}_{C=0} \int \mathrm{d}^{3} \boldsymbol{x}^{\prime \prime} \sqrt{\hat{\gamma}} /\left(-4 \pi r_{0}^{C}\left|\boldsymbol{x}-\boldsymbol{x}^{\prime \prime}\right|\right)$ on the " $r^{a}$ "-potential $\mathrm{FP}_{B=0} \int \mathrm{d}^{3} \boldsymbol{x}^{\prime} \sqrt{\hat{\gamma}}\left|\boldsymbol{x}-\boldsymbol{x}^{\prime}\right|^{a+B} f /\left(-4 \pi r_{0}^{B}\right)$ with $a \in \mathbb{Z}$. By permuting the two triple integrals, we obtain the relation

$$
\begin{array}{r}
\hat{\Delta}_{x}^{-1} \mathrm{FP}_{B=0} \int \frac{\mathrm{d}^{3} \boldsymbol{x}^{\prime} \sqrt{\hat{\gamma}}}{-4 \pi r_{0}^{B}}\left|\boldsymbol{x}-\boldsymbol{x}^{\prime}\right|^{a+B} f \\
=\mathrm{FP}_{B=0} \frac{\int \mathrm{d}^{3} \boldsymbol{x}^{\prime} \sqrt{\hat{\gamma} \mid} \boldsymbol{x}_{-\boldsymbol{x}^{\prime}(a+B+2} f}{-4 \pi r_{0}^{B}(a+B+2)(a+B+3)} .
\end{array}
$$

The result has the same form as the source. If we make $\hat{\Delta}^{-1}$ act on it, we arrive at a quantity of the same type. This provides a straightforward procedure to determine the action of $\hat{\Delta}^{-p}=$ $\left(\hat{\Delta}^{-1}\right)^{p}, p \in \mathbb{N}$, on the original integral iteratively. In this way, we find:

$$
\begin{aligned}
\hat{\Delta}_{x}^{-p} \mathrm{FP}_{B=0} \int \frac{\mathrm{d}^{3} \boldsymbol{x}^{\prime} \sqrt{\hat{\gamma}}}{-4 \pi r_{0}^{B}}\left|\boldsymbol{x}-\boldsymbol{x}^{\prime}\right|^{a+B} f \\
=\mathrm{FP}_{B=0} \int \frac{\mathrm{d}^{3} \boldsymbol{x}^{\prime} \sqrt{\hat{\gamma}}}{-4 \pi r_{0}^{B}}(a+B+1+2 p)^{-1} \\
\quad \times(a+B+2 p)^{-1} \ldots(a+B+2)^{-1} \\
\quad \times\left|\boldsymbol{x}-\boldsymbol{x}^{\prime}\right|^{a+B+2 p} f .
\end{aligned}
$$

If the usual " $r$ " "-potential with source $f$ exists, the $p$ th iterated Poisson integral $\hat{\Delta}_{x}^{-1}$ of its $p$ th derivative also does. It is equal to $\hat{\nabla}_{i_{1}} \ldots \hat{\nabla}_{i_{p}}$ applied to the right-hand side of Eq. (B.6). When we put all derivatives under the integration symbol, we end up with a convergent integral. At this stage, one no longer needs regularization, so we may take $B=0$ if none of the terms $(a+2 p+1),(a+2 p), \ldots,(a+2)$ vanish. Finally, we pull the coefficients $(a+1+2 p)^{-1}(a+2 p)^{-1} \ldots(a+1+p)^{-1}$ out of the integration symbol and reintroduce the finite part. We have then proved the formula giving the explicit action of $\hat{\Delta}_{x}^{-p}$ on arbitrary sources,

$$
\begin{gathered}
\hat{\Delta}_{x}^{-p} \mathrm{FP}_{B=0} \int \frac{\mathrm{d}^{3} \boldsymbol{x}^{\prime} \sqrt{\hat{\gamma}}}{-4 \pi r_{0}^{B}}\left|\boldsymbol{x}-\boldsymbol{x}^{\prime}\right|^{a+B} \hat{\nabla}_{i_{1}} \ldots \hat{\nabla}_{i_{p}} f= \\
\frac{\hat{\nabla}_{i_{1}} \ldots \hat{\nabla}_{i_{p}} \mathrm{FP}_{B=0} \int \frac{\mathrm{d}^{3} \boldsymbol{x}^{\prime} \sqrt{\hat{\gamma}}}{-4 \pi \pi_{0}^{8}}\left|\boldsymbol{x}-\boldsymbol{x}^{\prime}\right|^{a+B+2 p} f}{(a+1+2 p)(a+2 p) \ldots(a+2)},
\end{gathered}
$$

to be valid for $a+2 p+1 \notin \mathbb{N}$.

This allows us to write the potentials and super-potentials in a form suitable for numerical integration. For this purpose, 
we can recourse to fairly standard techniques, some of which were used in particular by Blanchet et al. (1990) to deal with the derivative of the Newtonian super-potential. With the help of Eq. (B.7) and the commutation relation $\hat{\Delta}_{x}^{-1} \hat{\nabla}_{i}=\hat{\nabla}_{i} \hat{\Delta}_{x}^{-1}$, we transform the expression of the (super-)potentials $\hat{\Delta}^{-2} \hat{\nabla}_{i} \hat{\nabla}_{j} F_{k l}$ or $\hat{\Delta}^{-3} \hat{\nabla}_{i} \hat{\nabla}_{j} \hat{\nabla}_{k} \hat{\nabla}_{l} F_{m n}$ entering the non-conformal part of the 3-metric (with possible index contractions). By doing this, we try to minimize the number of free indices that remain inside the integral and, for numerical reasons, to get rid of all spatial derivatives of the densities $D^{*}$ or $S_{i}^{*}$ in the final expressions. The transformation of $\hat{\Delta}^{-2} \hat{\nabla}_{i} \hat{\nabla}_{j} F_{k l}$, for instance, is achieved in four steps: (i) we let both derivatives commute with the integration symbol, $\hat{\Delta}^{-2} \hat{\nabla}_{i} \hat{\nabla}_{j} F_{k l}=\hat{\nabla}_{i} \hat{\nabla}_{j} \hat{\Delta}_{x}^{-2} F_{k l}$; (ii) we rewrite $\hat{\Delta}_{x}^{-2} F_{k l}$ according to Eq. (B.6) with $p=2$; (iii) we make one of the derivatives act on the kernel $\left|\boldsymbol{x}-\boldsymbol{x}^{\prime}\right|^{1+B}$ so that there remains only one unevaluated spatial derivative operating on a linear combination of simple Poisson integrals; and (iv) we let the derivative act. This yields

$$
\begin{array}{r}
\hat{\Delta}^{-2} \hat{\nabla}_{i} \hat{\nabla}_{j} F_{k l}=\frac{1}{2}\left[\hat{\gamma}_{i j} \hat{\Delta}_{\boldsymbol{x}}^{-1} F_{k l}+\hat{\gamma}_{j m} x^{m} \hat{\nabla}_{i} \hat{\Delta}_{\boldsymbol{x}}^{-1} F_{k l}\right. \\
\left.-\hat{\nabla}_{i} \hat{\Delta}_{\boldsymbol{x}}^{-1}\left(\hat{\gamma}_{j m} x^{m} F_{k l}\right)\right] .
\end{array}
$$

The respective transformation of $\hat{\Delta}^{-3} \hat{\nabla}_{i} \hat{\nabla}_{j} \hat{\nabla}_{k} F_{m n}$ is very similar:

$$
\begin{aligned}
\hat{\Delta}^{-3} \hat{\nabla}_{i} & \hat{\nabla}_{j} \hat{\nabla}_{k} F_{m n}=\frac{1}{24} \hat{\nabla}_{i} \hat{\nabla}_{j} \hat{\nabla}_{k} \mathrm{FP}_{B=0} \int \frac{\mathrm{d}^{3} \boldsymbol{x}^{\prime} \sqrt{\hat{\gamma}}}{-4 \pi r_{0}^{B}}\left|\boldsymbol{x}-\boldsymbol{x}^{\prime}\right|^{3+B} F_{m n} \\
= & \frac{1}{8} \hat{\nabla}_{k} \int \frac{\mathrm{d}^{3} \boldsymbol{x}^{\prime} \sqrt{\hat{\gamma}}}{-4 \pi}\left[\hat{\gamma}_{i p} \hat{\gamma}_{j q} \frac{\left(x^{p}-x^{\prime p}\right)\left(x^{q}-x^{\prime q}\right)}{\left|\boldsymbol{x}-\boldsymbol{x}^{\prime}\right|}\right. \\
& \left.+\hat{\gamma}_{i j}\left|\boldsymbol{x}-\boldsymbol{x}^{\prime}\right|\right] F_{m n} \\
=\frac{1}{8}[ & 3 \hat{\gamma}_{(i j} \hat{\gamma}_{k) p} x^{p} \hat{\Delta}_{\boldsymbol{x}}^{-1} F_{m n}-3 \hat{\gamma}_{(i j} \hat{\gamma}_{k) p} \hat{\Delta}_{\boldsymbol{x}}^{-1}\left(x^{p} F_{m n}\right) \\
& -2 \hat{\gamma}_{p(i} \hat{\gamma}_{j) q} x^{p} \hat{\nabla}_{k} \hat{\Delta}_{\boldsymbol{x}}^{-1}\left(x^{q} F_{m n}\right) \\
& +\hat{\gamma}_{i p} \hat{\gamma}_{j q} x^{p} x^{q} \hat{\nabla}_{k} \hat{\Delta}_{\boldsymbol{x}}^{-1} F_{m n} \\
& \left.+\hat{\gamma}_{i p} \hat{\gamma}_{j q} \hat{\nabla}_{k} \hat{\Delta}_{x}^{-1}\left(x^{p} x^{q} F_{m n}\right)\right] .
\end{aligned}
$$

We are now able to deduce the expression for $h_{i j}^{\mathrm{TT}}$ up to second post-Newtonian order, parametrized by means of the intermediate elementary potentials $\mathcal{S}, \mathcal{S}_{i}, \mathcal{T}_{i}, \mathcal{R}_{i}$, and $\mathcal{S}_{i j}$ as

$$
\begin{aligned}
h_{i j}^{\mathrm{TT}}= & \frac{1}{2} \mathcal{S}_{i j}-3 x^{k} \hat{\nabla}_{(i} \mathcal{S}_{j) k}+\frac{5}{4} \hat{\gamma}_{j m} x^{m} \hat{\nabla}_{i}\left(\hat{\gamma}^{k l} \mathcal{S}_{k l}\right) \\
& +\frac{1}{4} x^{k} x^{l} \hat{\nabla}_{i} \hat{\nabla}_{j} \mathcal{S}_{k l}+3 \hat{\nabla}_{(i} \mathcal{S}_{j)}-\frac{1}{2} x^{k} \hat{\nabla}_{i} \hat{\nabla}_{j} \mathcal{S}_{k} \\
& +\frac{1}{4} \hat{\nabla}_{i} \hat{\nabla}_{j} \mathcal{S}-\frac{5}{4} \hat{\nabla}_{i} \mathcal{T}_{j}-\frac{1}{4} \hat{\nabla}_{i} \mathcal{R}_{j} \\
& +\hat{\gamma}_{i j}\left[\frac{1}{4} \hat{\gamma}^{k l} \mathcal{S}_{k l}+x^{k} \hat{\gamma}^{l m} \hat{\nabla}_{m} \mathcal{S}_{k l}-\hat{\gamma}^{k l} \hat{\nabla}_{k} \mathcal{S}_{l}\right]+O\left(\frac{1}{c^{6}}\right) .
\end{aligned}
$$

This is the expression we use in Eq. (35) in Sect. 2.4 with the potentials determined by the scalar/vector/tensor Poisson Eqs. (30)-(34).

\section{Appendix C: multipole expansion of the intermediate potentials}

Here we derive the multipole expansion of the intermediate potentials $\mathcal{S}, \mathcal{S}_{i}, \mathcal{T}_{i}, \mathcal{R}_{i}$, and $\mathcal{S}_{i j}$ needed for obtaining boundary conditions for these potentials. This is done by means of the formula (C.9) of Blanchet \& Poujade (2002) specializing the matching relation first established in Blanchet (1998) for retarded quantities.

For any generic source $f$ which admits outside the system a multipole expansion of the form $\mathcal{M}(f)=\sum_{p=-\infty}^{p_{0}} f_{p}(\boldsymbol{n}) r^{p}$ with $\boldsymbol{n}=\boldsymbol{x} / r$ and $p_{0}<-2$, the multipole expansion of the Poisson integral $\hat{\Delta}^{-1} f$ is given by

$$
\begin{aligned}
\mathcal{M}\left(\hat{\Delta}^{-1} f\right)= & \sum_{l=0}^{+\infty} \frac{(-1)^{l}}{l !} \hat{\nabla}_{i_{1}} \ldots \hat{\nabla}_{i_{l}}\left(\frac{1}{r}\right) \\
& \times \mathrm{FP}_{B=0} \int \frac{\mathrm{d}^{3} x^{\prime} \sqrt{\hat{\gamma}}}{-4 \pi}\left(\frac{r^{\prime}}{r_{0}}\right)^{B} x^{\prime i_{1}} \ldots x^{\prime i_{l}} f \\
& +\hat{\Delta}_{\mathbf{0}}^{-1} \mathcal{M}(f) .
\end{aligned}
$$

In the special case where the source $f$ has compact support, $\mathcal{M}(f)$ is identically zero and thus the last term above vanishes. We recover the standard multipole formula used in electrostatics for spatially limited systems.

At this stage, the multipole expansions of all our elementary potentials may be derived by application of Eq. (C.1). We start with $\mathcal{S}_{i j}$ which goes to zero at the highest order. It involves the monopole integral with non-compact-supported source $\int \mathrm{d}^{3} \boldsymbol{x} \sqrt{\hat{\gamma}} \hat{\nabla}_{i} U \hat{\nabla}_{j} U /(-4 \pi)$. Remarkably, this integral admits an alternative expression whose source has compact support, which is a very useful feature for the numerical calculations. To perform the transformation, we first replace the second potential $U$ in the integrand $\hat{\nabla}_{i} U \hat{\nabla}_{j} U /(-4 \pi)$ by $\hat{\Delta} \chi / 2$, where $\chi$ denotes the Newtonian super-potential $\chi=\int \mathrm{d}^{3} \boldsymbol{x}^{\prime} \mid \boldsymbol{x}-$ $\boldsymbol{x}^{\prime} \mid D^{*}$. Next, we integrate this Laplacian by parts, being careful to keep all contributions from the derivatives of $r^{B}$. The result is

$$
\begin{aligned}
\mathrm{FP}_{B=} & \int \frac{\mathrm{d}^{3} \boldsymbol{x} \sqrt{\hat{\gamma}}}{-4 \pi r_{0}^{B}} r^{B} \hat{\nabla}_{i}\left(\frac{\chi}{2}\right) \hat{\nabla}_{j} \hat{\Delta} U \\
& +\mathrm{FP}_{B=0} \int \frac{\mathrm{d}^{3} \boldsymbol{x} \sqrt{\hat{\gamma}}}{-4 \pi r_{0}^{B}}\left[B(B+1) r^{B-2} \hat{\nabla}_{j} U\right. \\
& \left.+2 B r^{B-1} n^{k} \hat{\nabla}_{j} \hat{\nabla}_{k} U\right] \hat{\nabla}_{i}\left(\frac{\chi}{2}\right) .
\end{aligned}
$$

We remark here that the second finite part on the right-hand side vanishes. Indeed, the integration does not generate any pole in $B$ which is able to compensate for the cancellation of the pre-factor $B$ itself. Consequently, after a last integration by parts, we arrive at the equality

$$
\int \frac{\mathrm{d}^{3} \boldsymbol{x} \sqrt{\hat{\gamma}}}{-4 \pi} \hat{\nabla}_{i} U \hat{\nabla}_{j} U=-\frac{1}{2} \int \mathrm{d}^{3} \boldsymbol{x} \sqrt{\hat{\gamma}} D^{*} \hat{\nabla}_{i} \hat{\nabla}_{j} \chi .
$$

It is not difficult to check that $\hat{\nabla}_{j \chi}=\hat{\gamma}_{j k} x^{k} \hat{\Delta}^{-1}\left(-4 \pi D^{*}\right)-$ $\hat{\Delta}^{-1}\left(-4 \pi \hat{\gamma}_{j k} x^{k} D^{*}\right)$ by virtue of relation (B.7). Using an integration by parts of the type

$\int \mathrm{d}^{3} \boldsymbol{x} \sqrt{\hat{\gamma}} f \hat{\Delta}^{-1} g=\int \mathrm{d}^{3} \boldsymbol{x} \sqrt{\hat{\gamma}} g \hat{\Delta}^{-1} f$, 
we are finally able to show that

$$
\begin{aligned}
& \int \frac{\mathrm{d}^{3} \boldsymbol{x} \sqrt{\hat{\gamma}}}{-4 \pi} \hat{\nabla}_{i} U \hat{\nabla}_{j} U= \\
& \quad-\int \mathrm{d}^{3} \boldsymbol{x} \sqrt{\hat{\gamma}} D^{*}\left(\hat{\gamma}_{j k} x^{k} \hat{\nabla}_{i} U+\frac{1}{2} \hat{\gamma}_{i j} U\right) .
\end{aligned}
$$

Inserting the latter relation into the general formula (C.1) specialized for $f=-4 \pi S_{i}^{*} S_{j}^{*} / D^{*}-\hat{\nabla}_{i} U \hat{\nabla}_{j} U$, we get

$$
\begin{gathered}
\mathcal{M}\left(\mathcal{S}_{i j}\right)=\frac{1}{r} \int \mathrm{d}^{3} \boldsymbol{x} \sqrt{\hat{\gamma}} D^{*}\left(\frac{S_{i}^{*} S_{j}^{*}}{D^{* 2}}+\hat{\gamma}_{j k} x^{k} \hat{\nabla}_{i} U+\frac{1}{2} \hat{\gamma}_{i j} U\right) \\
+\mathcal{M}\left(-4 \pi \frac{S_{i}^{*} S_{j}^{*}}{D^{*}}-\hat{\nabla}_{i} U \hat{\nabla}_{j} U\right) .
\end{gathered}
$$

When examining the second term in the above equation, one immediately observes that $\mathcal{M}\left(S_{i}^{*} S_{j}^{*} / D^{*}\right)=0$ as the source $\left(S_{i}^{*} S_{j}^{*} / D^{* 2}\right) D^{*}$ has compact support. Furthermore we notice that $\mathcal{M}\left(\hat{\nabla}_{i} U \hat{\nabla}_{j} U\right)=O\left(1 / r^{4}\right)$, hence $\hat{\Delta}_{\mathbf{0}}^{-1} \mathcal{M}\left(\hat{\nabla}_{i} U \hat{\nabla}_{j} U\right)=$ $O\left(1 / r^{2}\right)$ according to dimensional analysis. It may also be checked directly with the help of the Matthew formula $\hat{\Delta}_{\mathbf{0}}^{-1}\left(n^{<i_{1}} \ldots n^{i_{l}>} r^{a}\right)=n^{<i_{1}} \ldots n^{i_{l}>} r^{a+2} /[(a+2-l)(a+3+l)]$.

We go on by calculating $\mathcal{M}\left(\mathcal{S}_{i}\right)$. The part of its source with non-compact support essentially amounts to $x^{k} \hat{\nabla}_{i} U \hat{\nabla}_{k} U$. The integral of the latter quantity may be transformed with the same technique as the one used to establish Eq. (C.6). We find that

$$
\begin{aligned}
\mathrm{FP}_{B=0} \int \frac{\mathrm{d}^{3} \boldsymbol{x} \sqrt{\hat{\gamma}}}{-4 \pi r_{0}^{B}} r^{B} x^{k} \hat{\nabla}_{i} U \hat{\nabla}_{k} U \\
=\frac{1}{2} \mathrm{FP}_{B=0} \int \frac{\mathrm{d}^{3} \boldsymbol{x} \sqrt{\hat{\gamma}}}{-4 \pi r_{0}^{B}} r^{B}\left(x^{k} \hat{\nabla}_{k} \chi \hat{\nabla}_{i}\left(-4 \pi D^{*}\right)\right. \\
\left.+2 \gamma^{k} l \hat{\nabla}_{k} \chi \hat{\nabla}_{l} \hat{\nabla}_{i} U\right) .
\end{aligned}
$$

We may then perform an integration by parts affecting the derivative $\hat{\nabla}_{i} D^{*}$ of the first term and the derivative $\hat{\nabla}_{k} \chi$ of the second term. The contribution of $\hat{\nabla}_{k} r^{B}$ is proportional to $B$. It can give rise to a definite non-zero result only when it is multiplied by the factor $1 / B$ coming from the radial integration of $1 / r^{3}$. As the corresponding angular integral vanishes, so does it. Noticing that $\hat{\Delta} \chi \hat{\nabla}_{i} U=2 U \hat{\nabla}_{i} U=\hat{\nabla}_{i} U^{2}$, we see that the resulting integral reads

$$
\begin{aligned}
& \frac{1}{2} \int \mathrm{d}^{3} \boldsymbol{x} \sqrt{\hat{\gamma}} D^{*}\left(x^{k} \hat{\nabla}_{i} \hat{\nabla}_{k \chi} \chi+\hat{\nabla}_{i} \chi\right) \\
& -\mathrm{FP}_{B=0} \int \mathrm{d}^{3} \boldsymbol{x} \sqrt{\hat{\gamma}}\left(\frac{r}{r_{0}}\right)^{B} \hat{\nabla}_{i} U^{2} .
\end{aligned}
$$

After integrating the second term by parts, we get an integral whose source is proportional to $B$. Following the same argument as before, it can be shown to be simply zero. Expressing again the super-potential as a combination of Poisson integrals, and performing other integrations by parts including those of the form (C.4), we arrive at

$$
\begin{aligned}
& \mathrm{FP}_{B=0} \int \frac{\mathrm{d}^{3} \boldsymbol{x} \sqrt{\hat{\gamma}}}{-4 \pi r_{0}^{B}} r^{B} x^{k} \hat{\nabla}_{i} U \hat{\nabla}_{k} U= \\
& -\int \mathrm{d}^{3} \boldsymbol{x} \sqrt{\hat{\gamma}} D^{*} \hat{\gamma}_{i j}\left(x^{j} U+x^{j} x^{k} \hat{\nabla}_{k} U\right) .
\end{aligned}
$$

On the other hand, the source $x^{k} \hat{\nabla}_{i} U \hat{\nabla}_{k} U$ behaves as $M^{2} \hat{\gamma}_{i k} n^{k} / r^{3}=-M^{2} \hat{\nabla}_{i}\left(r^{-2} / 2\right)$, where $M$ is the baryonic rest mass density defined in Sect. 2.4. A direct integration leads to the relation $\hat{\Delta}_{\mathbf{0}}^{-1}\left(1 / r^{2}\right)=\ln r+$ const., which can also be seen from a dimensional argument, so that $\hat{\Delta}_{\mathbf{0}}^{-1} \mathcal{M}\left(x^{k} \hat{\nabla}_{i} U \hat{\nabla}_{k} U\right)$ decreases asymptotically as $-M^{2} \hat{\nabla}_{i}(\ln r) / 2$. Therefore, we have proved the approximate equality

$$
\begin{gathered}
\mathcal{M}\left(\mathcal{S}_{i}\right)=\frac{1}{r} \int \mathrm{d}^{3} \boldsymbol{x} \sqrt{\hat{\gamma}} D^{*}\left[x^{k} S_{i} S_{k}+\hat{\gamma}_{i j} x^{j}\left(U+x^{k} \hat{\nabla}_{k} U\right)\right] \\
+\frac{M^{2}}{2 r} \hat{\gamma}_{i k} n^{k}+O\left(\frac{1}{r^{2}}\right) .
\end{gathered}
$$

In the multipole expansion of $\mathcal{T}_{i}$, the only term entering its composition that is generated by a quantity with noncompact support is the Poisson potential $\hat{\Delta}^{-1}\left(\hat{\gamma}_{i j} \hat{\gamma}^{k l} x^{j} \hat{\nabla}_{k} U \hat{\nabla}_{l} U\right)$. Its monopole part is composed of an integral over the source, which simplifies by virtue of the relation $2 \hat{\gamma}^{k l} \hat{\nabla}_{k} U \hat{\nabla}_{l} U=\hat{\Delta} U^{2}-$ $2 U \hat{\Delta} U$ to

$$
\begin{gathered}
\mathrm{FP}_{B=0} \int \frac{\mathrm{d}^{3} \boldsymbol{x}}{-4 \pi} \sqrt{\hat{\gamma}}\left(\frac{r}{r_{0}}\right)^{B}\left(\hat{\gamma}_{i j} \hat{\gamma}^{k l} x^{j} \hat{\nabla}_{k} U \hat{\nabla}_{l} U\right) \\
=-\int \mathrm{d}^{3} \boldsymbol{x} \sqrt{\hat{\gamma}} D^{*} \hat{\gamma}_{i k} x^{k} U,
\end{gathered}
$$

and of a contribution originating from the Poisson inverse operator applied to $\mathcal{M}\left(\hat{\gamma}_{i j} \hat{\gamma}^{k l} x^{j} \hat{\nabla}_{k} U \hat{\nabla}_{l} U\right)=-M^{2} \hat{\nabla}_{i}\left(r^{-2} / 2\right)+$ $O\left(1 / r^{4}\right)$. We thus have

$\hat{\Delta}_{\mathbf{0}}^{-1} \mathcal{M}\left(\hat{\gamma}_{i j} \hat{\gamma}^{k l} x^{j} \hat{\nabla}_{k} U \hat{\nabla}_{l} U\right)=-\frac{M^{2}}{2 r} \hat{\gamma}_{i j} n^{j}+O\left(\frac{1}{r^{2}}\right)$.

The treatment of the leading term in the multipole expansion of $\mathcal{R}_{i}$ is similar. The integral of $\hat{\nabla}_{i}\left(x^{k} x^{l} \hat{\nabla}_{k} U \hat{\nabla}_{l} U\right)$ is simply zero by virtue of the Gauss theorem, and the monopole part of $-\hat{\Delta}^{-1} \hat{\nabla}_{i}\left(x^{k} x^{l} \hat{\nabla}_{k} U \hat{\nabla}_{l} U\right)$ is the same as that of $2 \hat{\gamma}_{i j} \hat{\gamma}^{k l} x^{j} \hat{\nabla}_{k} U \hat{\nabla}_{l} U$.

We conclude with the multipole expansion for the potential $\mathcal{S}$. Since its source is already of compact support, it can simply be expanded with the help of the standard multipole formula mentioned after Eq. (C.1).

In the end this yields the desired multipole expansions of the elementary potentials in the second second post-Newtonian expansion, which correspond to Eqs. (36)-(40) in Sect. 2.4.

\section{Appendix D: post-Newtonian and post-Minkowskian link for gravitational waves}

The equation for null outgoing geodesics in the ADM gauge reads $t-r / c-2 M_{\mathrm{ADM}} G \ln [r /(c \eta)] / c^{3}+O\left(G^{2}\right)=$ const., with $M_{\mathrm{ADM}}=M+O\left(1 / c^{2}\right)$ being the $\mathrm{ADM}$ mass and $\eta$ an arbitrary positive constant with the dimension of time. For $r$ large enough compared to the typical wavelength, this also gives the link between the time $t$ and the distance $r$ of a field point $\boldsymbol{x}$ located near $\mathcal{I}^{+}$. In the post-Newtonian framework, $r / c$ is regarded as a small quantity (with respect to $t$ ), which precisely reflects the fact that the post-Newtonian metric is not accurate in the wave zone. Therefore, the expression (35) for $h_{i j}^{\mathrm{TT}}$ cannot be used there. Instead, we should consider the postMinkowskian version of Eq. (A.15), where all quantities are expanded in powers of the gravitational constant $G$. Higher order terms in $1 / c^{2}$ must not be neglected a priori, as long as they appear at a level of approximation below the one we want to reach. 
In general, this approach is not guaranteed to lead to a well-defined perturbative scheme in the ADM gauge in the sense that the contribution of order $G^{n+1}$ may become greater than the contribution of order $G^{n}$ in the neighborhood of $\mathcal{I}^{+}$. It is worthwhile briefly pointing out the origin of the possible problem. Once the field $h_{n_{i j}}^{\mathrm{TT}}$ is known at the $n$th postMinkowskian ( $n \mathrm{PM})$ level, the next approximation $h_{n+1} \mathrm{TT}$ is determined by the wave-like equation of the type $\hat{\square} h_{n+1}{ }_{i j}^{\mathrm{TT}}=$ $\hat{\gamma}_{i j}^{\mathrm{TT} k l} \Lambda_{n+1 k l}\left(h^{\mathrm{TT}}\right)+O\left(G^{n+2}\right)$ obtained by combing Eqs. (A.4) and (A.5) as explained in Appendix A. The source $\Lambda_{n+1 i j}$ depends non-linearly on $h_{i j}^{\mathrm{TT}}$, which can thus be replaced by its $n \mathrm{PM}$ value $h_{n k l}^{\mathrm{TT}}$. In the end, the solution of the previous wave equation is the retarded integral of $\hat{\gamma}_{i j}^{\mathrm{TT} k l} \Lambda_{n+1 k l}\left(h^{\mathrm{TT}}\right)$, which we denote in short as $\hat{\Delta}^{-1} \hat{\gamma}_{i j}^{\mathrm{TT} k l} \Lambda_{n+1 k l}$. The transverse traceless projector can itself be written as a converging integral with the help of a modified version of Eq. (B.7), in which the derivatives are applied to the kernel $\left|\boldsymbol{x}-\boldsymbol{x}^{\prime}\right|^{\alpha+B+2 p}$ with $\alpha=-1$. Even if the retarded integral converges, it may be conveniently replaced by its Hadamard finite part. The numerical result is not affected, but the integral appearing under the operator $\mathrm{FP}_{B=0}$ can now be shown to commute with the integral defining $h_{n+1 i j}$ TT after an appropriate analytic continuation. As a consequence, the finite part of $\hat{\gamma}_{i j}^{\mathrm{TT} k l}$ (regarded as an integral operator, which may be referred to as $\hat{\gamma}_{i j}^{\mathrm{TT} k l}$ without any ambiguity) can be pulled out from the d'Alembertian inverse symbol: $\hat{\square}^{-1} \hat{\gamma}_{i j}^{\mathrm{TT} k l} \Lambda_{n+1 k l}=\hat{\gamma}_{i j}^{\mathrm{TT} k l} \hat{\square}^{-1} \Lambda_{n+1 k l}$. The behavior of $\Lambda_{n+1 i j}\left(h_{n}{ }^{\mathrm{TT}}\right)$ when $r \rightarrow+\infty$ follows from a formula extending Eq. (C.1) to the case where the quantity to be calculated is a retarded integral. In particular, the multipole expansion of $h_{i j}^{\mathrm{TT}}$ involves the retarded integral of the multipole expansion of the source, regularized by means of the Hadamard finite part $\hat{a}_{0}^{-1} \mathcal{M}\left(\Lambda_{n+1 i j}\right)$. This contribution turns out to generate terms tending towards zero as $(\ln r)^{2} / r$ at the $2 \mathrm{PM}$ order, which are thus out of control. The structure of the field has not been investigated yet at higher PM order in ADM gauge, but a similar phenomenon is likely to happen in that case too.

Fortunately, the previous problem can be cured at the postlinear level either by moving to some radiative gauge where $u=$ $t-r / c$ is a conformal coordinate, or by absorbing the logarithms into the arguments of some multipole moments. In harmonic gauge, the logarithms occurring in the post-Minkowskian iteration were shown (Blanchet 1987) to be removable to all orders. In ADM gauge, the possibility of such an elimination has been checked explicitly at the post-linear level by Schäfer (1990) from a formula adapting the derivation of Appendix A in order to include in the Hamiltonian the relative Newtonian part of the 2PM corrections to all terms quadratic in $h_{i j}^{\mathrm{TT}}\left(\right.$ or $\left.\pi_{\mathrm{TT}}^{i j}\right)$. The new wave equation for the field variable reads

$$
\begin{aligned}
\hat{\square} h_{i j}^{\mathrm{TT}}= & \gamma_{i j}^{\mathrm{TT} k l}\left[F_{k l}+\frac{4}{c^{2}}\left(-2 \pi G D^{*} h_{k l}^{\mathrm{TT}}\right.\right. \\
& \left.\left.+\hat{\gamma}^{m n} \hat{\nabla}_{m} U \hat{\nabla}_{n} h_{k l}^{\mathrm{TT}}+U \hat{\Delta} h_{k l}^{\mathrm{TT}}\right)\right] \\
& +O\left(\frac{\left(h^{\mathrm{TT}}\right)^{0}}{c^{6}}\right)+O\left(\frac{\left(h^{\mathrm{TT}}\right)^{1}}{c^{4}}\right)+O\left(\left(h^{\mathrm{TT}}\right)^{2}\right) .
\end{aligned}
$$

The Green function method provides the required solution, but the computation is rather delicate. The result is a retarded integral, to which it remains to apply the transverse tracefree projector $\hat{\gamma}_{i j}^{\mathrm{TT} k l}$. The action of the second spatial derivatives $\hat{\nabla}_{k} \hat{\nabla}_{l}$ on a quantity of the form $f(t-r / c) / r$ leads to the "monopole" term $\hat{\gamma}_{k p} \hat{\gamma}_{l q} n^{p} n^{q} \ddot{f}(t-r / c) /\left(r c^{2}\right)$, each dot denoting a time derivative, plus $1 / r^{2}$ corrections. It follows that $\hat{\Delta}^{-1} \hat{\nabla}_{k} \hat{\nabla}_{l}[f(t-r / c) / r]=\hat{\gamma}_{k p} \hat{\gamma}_{l q} n^{p} n^{q} f(t-r / c) / r+O\left(1 / r^{2}\right)$. Similarly, it is not difficult to check that $\hat{\Delta}^{-2} \hat{\nabla}_{i} \hat{\nabla}_{j} \hat{\nabla}_{k} \hat{\nabla}_{l}[f(t-$ $r / c) / r]=\hat{\gamma}_{i p} \hat{\gamma}_{j q} \hat{\gamma}_{k r} \hat{\gamma}_{l s} n^{p} n^{q} n^{r} n^{s} f(t-r / c) / r+O\left(1 / r^{2}\right)$. This implies that the operator (A.6), applied to a term $f(t-r / c) / r$, reduces to the projector $P_{i j}^{\mathrm{TTkl}}$. Using the latter property, we recover the well-known relation

$$
\begin{aligned}
h_{i j}^{\mathrm{TT}}= & \frac{1}{r} P_{i j}^{\mathrm{TT} k l} \\
& \times\left\{M _ { k l } \left[t-\frac{r}{c}-\frac{2 G}{c^{3}}\left(M+O\left(\frac{1}{c^{2}}\right)\right) \ln \left(\frac{r}{c \eta}\right)\right.\right. \\
& \left.\left.+O\left(G^{2}\right)\right]+O\left(\frac{1}{c^{2}}\right)\right\}+O\left(\frac{1}{r^{2}}\right),
\end{aligned}
$$

with $M_{i j}(t)=\int \mathrm{d}^{3} \boldsymbol{x} \sqrt{\hat{\gamma}} F_{i j} /(-4 \pi)$ being the Newtonian monopole moment associated to the source $F_{i j}$.

Because of the algebraic transverse traceless projection, we may substitute $M_{\langle i j\rangle}$ by $M_{i j}$ without affecting the outcome in the above relation. Since we still work at the $2 \mathrm{PN}$ level, we do not need to include any higher order corrections in the expression of $M_{i j}$. The retarded argument $t-r / c-2 G M_{\mathrm{ADM}} / c^{3} \ln [r /(c \eta)]+$ $O\left(G^{2}\right)$ coincides with the null radiative coordinate. It can be viewed as the actual retarded time $t_{\text {ret }}$ of the field variable at $\mathcal{I}^{+}$up to the 1PM order. Taking the preceding into account, we arrive at

$h_{i j}^{\mathrm{TT}} \approx P_{i j}^{\mathrm{TT} k l} \frac{M_{<k l>}\left(t_{\mathrm{ret}}\right)}{r}+O\left(\frac{1}{r^{2}}\right)$.

We now improve the formula for $M_{i j}$ by making use of Eq. (C.5), which leads to

$M_{\langle i j\rangle}=\frac{4 G}{c^{4}} \int \mathrm{d}^{3} \boldsymbol{x} \sqrt{\hat{\gamma}} D^{*}\left(\frac{S_{\langle i}^{*} S_{j\rangle}^{*}}{D^{* 2}}+x^{k} \hat{\gamma}_{k<i} \hat{\nabla}_{j\rangle} U\right)$.

Excluding 1PN errors, this integral equals $4 G \int \mathrm{d}^{3} \boldsymbol{x} \sqrt{\hat{\gamma}} D\left(\hat{\gamma}_{k i} \hat{\gamma}_{l j} v^{\langle k} v^{l\rangle}+x^{k} \hat{\gamma}_{k\langle i} \hat{\nabla}_{j\rangle} U\right) / c^{4}$. This is proportional to (twice) the second time derivative of the Newtonian quadrupole $2 \ddot{Q}_{i j}$ at this level, as can be checked with the help of the relation

$$
\begin{aligned}
& \frac{\mathrm{d}}{\mathrm{d} t} \int \mathrm{d}^{3} \boldsymbol{x} \sqrt{\hat{\gamma}} D^{*} f(\boldsymbol{x}, t)= \\
& \int \mathrm{d}^{3} \boldsymbol{x} \sqrt{\hat{\gamma}} D^{*}\left(v^{i} \hat{\nabla}_{i}+\partial_{t}\right) f(\boldsymbol{x}, t),
\end{aligned}
$$

which is valid for any $C^{1}$ function $f$. We have thus derived the Newtonian quadrupole formula

$h_{i j}^{\mathrm{TT}} \sim P_{i j}^{\mathrm{TT} k l} \frac{2 G \ddot{Q}_{k l}}{r c^{4}}$

in the neighborhood of future null infinity. 
It is now possible to investigate the asymptotic behavior of $h_{i j}^{2 \mathrm{PN}}$ in a similar way as we did for the intermediate potentials. The computation does not present any particular subtleties in contrast to that of the full field variable dominant contribution. The operator $\hat{\gamma}_{i j}^{\mathrm{TT} k l}$ commutes with the Poisson integral so that $\mathcal{M}\left(\hat{\Delta}^{-1} \hat{\gamma}_{i j}^{\mathrm{TT} k l} F_{k l}\right)=\hat{\gamma}_{i j}^{\mathrm{TT} k l} \mathcal{M}\left(\hat{\Delta}^{-1} F_{k l}\right)=\hat{\gamma}_{i j}^{\mathrm{TT} k l}\left(M_{k l} / r\right)+$ $O\left(1 / r^{2}\right)$. The action of the transverse trace-free projector is computed with the help of Eq. (B.4), which gives

$$
\begin{gathered}
h_{i j}^{2 \mathrm{PN}}(\boldsymbol{x}, t)=\left[\frac{3}{16} \hat{\gamma}_{(i j} \hat{\gamma}^{k l)}+\frac{3}{4} \delta_{(i}^{k} \hat{\gamma}_{j) p} n^{l} n^{p}\right. \\
+\frac{3}{16} \hat{\gamma}_{i p} \hat{\gamma}_{j q} n^{p} n^{q} n^{k} n^{l} \\
\left.-\frac{5}{16}\left(\hat{\gamma}_{i j} n^{k} n^{l}+\hat{\gamma}^{k l} \hat{\gamma}_{i p} \hat{\gamma}_{j q} n^{p} n^{q}\right)\right] \\
\times \frac{M_{k l}(t)}{r}+O\left(\frac{1}{r^{2}}\right),
\end{gathered}
$$

with $M_{\langle i j\rangle}=2 G \ddot{Q}_{i j} / c^{4}$. By applying the operator $P_{i j}^{\mathrm{TT} k l}$ on both sides of Eq. (D.7), we find that $P_{i j}^{\mathrm{TT} k l} h_{k l}^{2 \mathrm{PN}}=P_{i j}^{\mathrm{TT} k l} G \ddot{Q}_{k l} /\left(4 r c^{4}\right)$. As a conclusion, with the notations of Sect. 3.3, we have (cf. Eqs. (52), (53)):

$P_{i j}^{\mathrm{TT} k l} h_{k l}^{2 \mathrm{PN}}\left(\boldsymbol{x}, t_{\mathrm{ret}}\right) \sim \frac{1}{8} h_{i j}^{\mathrm{quad}}(\boldsymbol{x}, t)$.

\section{References}

Arnowitt, R., Deser, S., \& Misner, C. 1962, in Gravitation: An introduction to current research, ed. L. Witten (New York, USA: Wiley), 227

Baiotti, L., Hawke, I., Montero, P., et al. 2004, Phys. Rev. D, in press [gr-qc/0403029]

Banyuls, F., Font, J., Ibáñez, J., Martí, J., \& Miralles, J. 1997, ApJ, 476, 221

Blanchet, L. 1987, Proc. R. Soc. London A, 409, 383

Blanchet, L. 1998, Class. Quantum Grav., 15, 1971

Blanchet, L., \& Damour, T. 1986, Philos. Trans. R. Soc. London A, 320,379

Blanchet, L., \& Poujade, O. 2002, Phys. Rev. D, 65, 124020

Blanchet, L., Damour, T., \& Schäfer, G. 1990, MNRAS, 242, 289

Bonazzola, S., \& Marck, J. 1993, A\&A, 267, 623

Colella, P., \& Woodward, P. 1984, J. Comput. Phys., 54, 174

Dimmelmeier, H., Font, J., \& Müller, E. 2001, ApJ, 560, L163

Dimmelmeier, H., Font, J., \& Müller, E. 2002a, A\&A, 388, 917

Dimmelmeier, H., Font, J., \& Müller, E. 2002b, A\&A, 393, 523
Dimmelmeier, H., Novak, J., Font, J., Ibáñez, J., \& Müller, E. 2005, Phys. Rev. D, 71, 064023

Donat, R., \& Marquina, A. 1996, J. Comput. Phys., 125, 42

Duez, M., Baumgarte, T., Shapiro, S., Shibata, M., \& Uryu, K. 2002, Phys. Rev. D, 65, 024016

Finn, L. 1989, in Frontiers in numerical relativity, ed. C. Evans, S. Finn, \& D. Hobill (Cambridge, UK: Cambridge University Press), 126

Font, J. 2003, Living Rev. Relativity, 6, 4, (Online article): cited on http: //www . livingreviews .org//rr-2003-4

Font, J., Goodale, T., Iyer, S., et al. 2002, Phys. Rev. D, 65, 084024

Font, J., Stergioulas, N., \& Kokkotas, K. 2000, MNRAS, 313, 678

Fryer, C., Holz, D., \& Hughes, S. 2002, ApJ, 565, 430

Fryer, C., Holz, D., Hughes, S., \& Warren, M. 2004, in Stellar Collapse, ed. C. Fryer, Astrophysics and Space Science Library (Amsterdam, The Netherlands: Kluwer Academic), 373

Holm, C. 1985, Physica, 17D, 1

Imamura, J., Pickett, B., \& Durisen, R. 2003, ApJ, 587, 341

Isenberg, J. 1978, University of Maryland Preprint, unpublished

Janka, H.-T., Zwerger, T., \& Mönchmeyer, R. 1993, A\&A, 268, 360

Kley, W., \& Schäfer, G. 1999, Phys. Rev. D, 60, 027501

Komatsu, H., Eriguchi, Y., \& Hachisu, I. 1989a, MNRAS, 237, 355

Komatsu, H., Eriguchi, Y., \& Hachisu, I. 1989b, MNRAS, 239, 153

Kotake, K., Yamada, S., \& Sato, K. 2003, Phys. Rev. D, 68, 044023

Lattimer, J., \& Swesty, F. 1991, Nucl. Phys. A, 535, 331

Mönchmeyer, R., Schäfer, G., Müller, E., \& Kates, R. 1991, A\&A, 246, 417

Müller, E. 1982, A\&A, 114, 53

Müller, E., Rampp, M., Buras, R., Janka, H.-T., \& Shoemaker, D. 2004, ApJ, 603, 221

Müller, E., \& Steinmetz, M. 1995, Comp. Phys. Comm., 89, 45

Ott, C., Burrows, A., Livne, E., \& Walder, R. 2004, ApJ, 600, 834

Rampp, M., Müller, E., \& Ruffert, M. 1998, A\&A, 332, 969

Regge, T., \& Teitelboim, C. 1974, Ann. Phys., 88, 286

Schäfer, G. 1985, Ann. Phys. N.Y., 81, 161

Schäfer, G. 1990, Astron. Nachr., 311, 213

Schwinger, J. 1963, Phys. Rev., 130, 1253

Shibata, M., \& Sekiguchi, Y. 2004a, Phys. Rev. D, 69, 084024

Shibata, M., \& Sekiguchi, Y. 2004b, Phys. Rev. D, 71, 024014

Siebel, F., Font, J., Müller, E., \& Papadopoulos, P. 2003, Phys. Rev. D, 67, 124018

Wilson, J., Mathews, G., \& Marronetti, P. 1996, Phys. Rev. D, 54, 1317

Yamada, S., \& Sato, K. 1995, ApJ, 450, 245

Zwerger, T. 1995, Ph.D. Thesis, Technische Universität München,

München, Germany

Zwerger, T., \& Müller, E. 1997, A\&A, 320, 209 